\title{
A generalised model of judgment aggregation
}

\author{
Franz Dietrich ${ }^{1}$ \\ October 2004, last revised April 2006
}

\begin{abstract}
The new field of judgment aggregation aims to merge many individual sets of judgments on logically interconnected propositions into a single collective set of judgments on these propositions. Judgment aggregation has commonly been studied using classical propositional logic, with a limited expressive power and a problematic representation of conditional statements ("if $P$ then $Q$ ") as material conditionals. In this methodological paper, I present a simple unified model of judgment aggregation in general logics. I show how many realistic decision problems can be represented in it. This includes decision problems expressed in languages of classical propositional logic, predicate logic (e.g. preference aggregation problems), modal or conditional logics, and some multi-valued or fuzzy logics. I provide a list of simple tools for working with general logics, and I prove impossibility results that generalise earlier theorems.
\end{abstract}

Key words: judgment aggregation, discursive dilemma, modelling methodology, general logics, impossibility theorem

\section{Introduction}

The traditional model of social choice theory, preference aggregation, defines a collective decision problem as the problem of forming collective preferences over a given set of alternatives (actions, policies, candidates, states of society etc.). By contrast, the newly arising model of judgment aggregation defines a collective decision problem as the problem of forming collective judgments (acceptance or rejection) on a given set of logically interrelated propositions. As a simple example, suppose the population of a country disagrees on whether the following propositions hold: ${ }^{2}$

$a: \quad$ The birth rate is too low to guarantee long-term economic stability.

$b: \quad$ More immigration is needed.

$a \rightarrow b:$ If the birth rate is too low to guarantee long-term economic stability, then more immigration is needed.

Reaching collective judgments on logically interrelated propositions is non-trivial. Suppose that in the example the population is split into three camps of equal size.As

\footnotetext{
${ }^{1}$ This paper was presented at different occasions under the title "Judgment aggregation in general logics", before a referee kindly recommended the new title. Many thanks to Ruvin Gekker, Franz Huber, Philippe Mongin, Marc Pauly, Nobu-Yuki Suzuki, and two referees for most helpful advice. The paper greatly benefited from discussions with Christian List and extensive comments by Martin van Hees - warm thanks also to them. This research was supported by the Alexander von Humboldt Foundation, the Federal Ministry of Education and Research, and the Program for the Investment in the Future (ZIP) of the German Government.

${ }^{2}$ This example is taken from an unpublished opinion poll conducted by André Habisch, Christian List and René Schmidpeter, who find a similar collective inconsistency.
} 


\begin{tabular}{|l|c|c|c|}
\hline & $a$ & $a \rightarrow b$ & $b$ \\
\hline $1 / 3$ of population & True & True & True \\
\hline $1 / 3$ of population & True & False & False \\
\hline 1/3 of population & False & True & False \\
\hline Majority rule & True & True & False \\
\hline Premise-based rule & True & True & True \\
\hline Conclusion-based rule & & & False \\
\hline
\end{tabular}

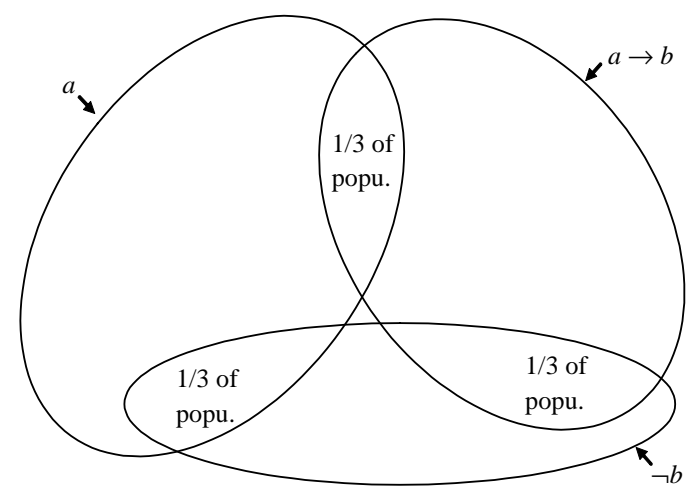

Figure 1: A discursive dilemma, shown as a table (left) and as a Venn diagram (right)

shown in Figure 1, each camp holds a consistent set of judgments; for instance the first camp accepts $a$ and $a \rightarrow b$, and accordingly accepts $b$. Yet the propositionwise majority rule results in an inconsistent set of judgments, namely $\{a, a \rightarrow b, \neg b\}$. Such situations are known as discursive dilemmas (e.g. Pettit 2001).

An agenda of judgment aggregation (i.e. a set of propositions under consideration) can be represented by a Venn diagram: each proposition is represented by a set of worlds in which the proposition is true, where an inconsistency between propositions is indicated by the non-overlap of their sets of worlds. For instance, in the Venn diagram of our example agenda (see Figure 1) the propositions $a, a \rightarrow b, \neg b$ have non-overlapping sets of worlds, i.e. in no world all of $a, a \rightarrow b, \neg b$ are true. ${ }^{3}$

How can the group reach consistent decisions in the face of a discursive dilemma? Two aggregation rules have received particular attention; let me define them for our example. Under the premise-based rule, the group takes majority votes only on $a$ and $a \rightarrow b$ (the premises) and decides $b$ (the conclusion) by entailment from the decisions on $a$ and $a \rightarrow b$. So, $a$ and $a \rightarrow b$ are accepted, and hence $b$ is accepted. Under the conclusion-based rule, the group takes a majority vote only on $b$, ignoring any majority verdicts on $a$ and $a \rightarrow b$. So $b$ is rejected and no collective judgment is made on $a$ and $a \rightarrow b$.

The propositions in judgment aggregation can be atomic (such as $a$ or $b$ ) or compound (such as $a \rightarrow b$ ), and they can express various things such as beliefs (e.g. "pollution creates global warming"), desires (e.g. "global warming is undesirable") and act preferences (e.g. "measure X against pollution should be taken"). Judgment aggregation is also close to real decision situations. First, individuals are not required to rank complex alternatives but only to have opinions on different issues (propositions). Second, real decision situations often consist indeed in having to accept or reject different interconnected propositions.

In this paper, I argue that judgment aggregation allows one to study a wide range of realistic collective decision problems provided that the model is extended beyond classical propositional logic. While classical propositional logic can adequately rep-

\footnotetext{
${ }^{3}$ The Venn diagram shown in Figure 1 already anticipates that " $\rightarrow$ " should not be a material conditional, by including worlds in which $a \rightarrow b$ is false without both of $a$ and $\neg b$ being true.
} 
resent decision problems whose propositions involve the logical connectives "and", "or", "not" (or other truth-functional connectives), most real problems do not take this simple form and require more expressive languages. Problems involving conditional statements like $a \rightarrow b$ above usually require a conditional logic, although they were so far represented in classical propositional logic by using the (problematic) material conditional, as explained later. ${ }^{4}$ Other realistic decision problems may be represented in a predicate logic, in which atomic propositions are not taken as primitives but are constructed from constants, variables, functions and relations (just as in common language sentences are not primitives). This allows for instance to embed preference aggregation into judgment aggregation. Many decision problems can be represented using modal logics, in which one can express propositional attitudes such as "it is desirable that $p$ ", "it is ethically required/allowed that $p$ ", "it is probable that $p$ " etc. Decision problems with vague propositions (like "equality in society is very important") may require a fuzzy logic.

The good news is that a unified model of judgment aggregation is still possible, despite of the differences between the logics necessary for the various applications. I introduce a model of "general logics" that is not restricted to any particular logic but allows most practically relevant logics. Several previous results, if suitably restated, can be shown to hold in general logics; many others are yet to be derived.

In Section 2, I introduce the model, which is based on a set of mild conditions on the logic. In Section 3, I illustrate the model by discussing several types of collective decision problems, all of which can be represented within the model. In Section 4, I provide a list of simple tools that can be used to prove results in general logics; these tools underlie many existing proofs in judgment aggregation, which shows that one can conveniently work in general logics. In Section 5, I prove an impossibility theorem, which generalises earlier results to general logics. In Section 6, I mention that it can also be interesting to derive results restricted to a particular logic (often not classical propositional logic), which leads me to contrast judgment aggregation in general logics with judgment aggregation in a particular logic. In Section 7, I summarise and conclude the paper.

On a less formal basis, judgment aggregation has been discussed already for a while, partly focussing on the distinction between premise-based and conclusionbased decision-making (e.g. Kornhauser and Sager 1986, Chapman 1998, 2002, Pettit 2001, Brennan 2001, Bovens and Rabinowicz 2004). List and Pettit (2002) formalised judgment aggregation in classical propositional logic, and proved a first social-choicetheoretic impossibility result. This sparked a series of contributions. Pauly and van Hees (2004), Dietrich (2004), Gärdenfors (2004), Nehring and Puppe (2004), van Hees (2004) and Mongin (2005) prove several impossibility theorems, whose main message is that, given certain logical connections between the propositions under decision, propositionwise aggregation (satisfying some mild conditions) is impossible. Several important impossibility results follow from Nehring and Puppe's (2002) theorems about property spaces. To escape impossibilities, one may for instance restrict the domain of the aggregation rule (List 2003), restrict the independence condition to premises (Dietrich 2004), use fusion operators (Pigozzi 2004), or use sequential de-

\footnotetext{
${ }^{4}$ In the literature, a problematic use of material conditionals (by authors including myself) occurs mainly when giving examples of judgment aggregation. Most formal results do not use any conditionals; they are thus silent about decision problems like that of our immigration example.
} 
cision rules (List 2004b and Dietrich and List 2005). The probability of "correct" collective judgments is analysed by Bovens and Rabinowicz (2004) and List (2004a). Gärdenfors (2004) and Gekker (2003) question the requirement that judgments must be complete. Strategy-proof judgment aggregation is analysed in Dietrich and List (2004a). List and Pettit (2004) discuss the connection to preference aggregation.

The models of Pauly and van Hees (2004) and van Hees (2004) allow for degrees of acceptance, a significant generalisation of the informational input and output of decision rules, but still with the same limited expressive power of propositions as in the standard model. The difference between their model generalisation and the one undertaken here is that they generalise the attitude towards propositions, whereas I generalise what propositions can express. Their models use multi-valued logics. Also in the present model the propositions may belong to a multi-valued (or fuzzy) logic, but even then what is aggregated are sets of judgments, not entire valuation functions. ${ }^{5}$

\section{A judgment aggregation model in general logics}

\subsection{Why judgment aggregation needs logic}

Usually, a logic is defined by (i) certain rules for constructing propositions (statements), (ii) a notion of (semantic) entailment that specifies when a set of propositions $A$ entails a proposition $p$ (if $A$ entails $p$, we write $A \vDash p$ ), and (iii) a notion of (syntactic) derivability/provability that specifies rules for how to derive/prove a proposition $p$ from a set of proposition $A$ (if $p$ is derivable from $A$, we write $A \vdash p$ ). The rules for deriving/proving are complete and sound if all propositions entailed by a set, and no other propositions, are derivable from the set (i.e. $A \vDash p$ if and only if $A \vdash p$ ), as it is the case for classical propositional or predicate logic.

In judgment aggregation, we need a logical framework in order to define rationality notions, notably the notions of consistency and deductive closure of sets of judgments. Depending on whether one holds a semantic or a syntactic concept of rationality, one might choose to define these notions in terms of entailment or in terms of derivability. In particular, in the first case a set of judgments is consistent if it does not entail a contradiction, and in the second case it is consistent if no contradiction can be derived/proven from it. Being rational in a semantic or a syntactic sense differs from each other in a roughly similar way as "believing what is true" differs from "believing what one can prove" (which in turn resembles the distinction between true beliefs and justified beliefs). Interpretational differences aside, semantic and syntactic rationality notions may of course become formally identical if the rules of derivation are complete and sound (see above): then a set of judgments is consistent (respectively deductively closed) in the semantic sense if and only if it is so in the syntactic sense.

\subsection{General logics}

Whether we want to define the rationality notions semantically or syntactically, all we need is the entailment relation $\vDash$ (respectively derivability relation $\vdash$ ) between

\footnotetext{
${ }^{5}$ For a multi-valued or fuzzy logic (with more than two truth values), a valuation function contains more information than a set of judgments: it gives a truth-value to each proposition, rather than just telling which propositions are "accepted".
} 
sets of propositions $A$ and propositions $p$. Thus the model will leave open how this relation is itself generated: the model does not specify any particular way to define $\vDash$, say in terms of truth-functions or interpretations (respectively any particular rules of deduction to define $\vdash$ ). This is why I speak of "general" logics.

Moreover, we need only one of the two relations: only $\vDash$ if we go semantically and only $\vdash$ if we go syntactically. Thus our general logic is in fact only half a logic, namely either a "general semantic" or a "general syntax". Hence I will use a single relation, which may be interpreted either as entailment $\vDash$ or as derivability $\vdash$ (as I said, depending on one's rationality concept). Despite of allowing both interpretations, I will use the symbol $\vDash$ and speak of "entailment", because I tend to find semantic rationality notions more natural for the purpose of judgment aggregation. ${ }^{6}$

So let me define a logic or formal language (with negation $\neg$ ) as a pair $(\mathbf{L}, \models)$ consisting of

- a non-empty set $\mathbf{L}$ of formal expressions ${ }^{7}$, called propositions, such that $p \in \mathbf{L}$ implies $\neg p \in \mathbf{L}$;

- a binary relation $\vDash(\subseteq \mathcal{P}(\mathbf{L}) \times \mathbf{L})$ between sets $A \subseteq \mathbf{L}$ and propositions $p \in \mathbf{L}$, called the entailment relation; $A \vDash p$ is read " $A$ entails $p$ " or " $p$ follows from $A^{\prime \prime} ;$ I write $p_{1}, \ldots, p_{k} \vDash p$ for $\left\{p_{1}, \ldots, p_{k}\right\} \vDash p$.

While $\mathbf{L}$ tells what propositions can be formed in the language, $\vDash$ tells how they are logically interrelated. Inconsistency can be defined out of entailment:

Definition 1 (a) $A$ set $A \subseteq \mathbf{L}$ is inconsistent if there is a $p \in \mathbf{L}$ such that $A \vDash p$ and $A \vDash \neg p$, and consistent otherwise.

(b) A proposition $p \in \mathbf{L}$ is a contradiction if $\{p\}$ is inconsistent, a tautology if $\{\neg p\}$ is inconsistent, and contingent if $\{p\}$ and $\{\neg p\}$ are consistent.

This abstract notion of a logic is very flexible, as examples will illustrate. All basic notions of judgment aggregation can be defined for a general logic $(\mathbf{L}, \vDash)$ in the familiar way: agendas, judgment sets, rationality conditions (e.g. deductive closure), aggregation rules, and conditions on aggregation rules (anonymity, independence, etc.).

To obtain interesting results such as (im)possibility theorems, some conditions on the logic $(\mathbf{L}, \models)$ are of course inevitable. The good news is that three mild conditions are often sufficient (and necessary): ${ }^{8}$

$\mathbf{L} 1$ For any $p \in \mathbf{L}, p \vDash p$ (self-entailment).

L2 For any $p \in \mathbf{L}$ and $A \subseteq B \subseteq \mathbf{L}$, if $A \vDash p$ then $B \vDash p$ (monotonicity).

L3 The empty set $\emptyset$ is consistent, and each consistent set $A \subseteq \mathbf{L}$ has a consistent superset $B \subseteq \mathbf{L}$ containing a member of each pair $p, \neg p \in \mathbf{L}$ (completability).

Under L1-L3, any proposition entails itself, any entailment is preserved by adding new premises, and any consistent set can be extended to a complete consistent set.

\footnotetext{
${ }^{6}$ Derivations have for me more the (epistemic) status of helping to ascertain the rationality of a set of judgments, not of defining it.

${ }^{7} \mathrm{~A}$ formal expression is any (finitely long) concatenation of symbols, such as $a$ or $c \rightarrow x$ or $\forall x(P x)$ or $(((s+3=\% \$$.

${ }^{8}$ Monotonicity is a standard term in logic (unlike self-entailment and completability). So-called algebraic logic investigates abstract conditions on the derivability relation $\vdash$ similar to L1-L5, as I learned from Nobu-Yuki Suzuki.
} 
Most realistic judgment aggregation problems can be formalised in logics of type L1-L3 (see Section 2). While many arguments commonly used in proofs work perfectly for a general logic $(\mathbf{L}, \vDash)$ of type L1-L3, some arguments require additional properties, which are also satisfied by the logics of many realistic aggregation problems:

L4 For any $A \subseteq \mathbf{L}$ and $p \in \mathbf{L}$, if $A \cup\{\neg p\}$ is inconsistent then $A \vDash p$ (nonparaconsistency). ${ }^{9}$

L5 For any $p \in \mathbf{L}$ and $A \subseteq \mathbf{L}$, if $A \vDash p$ then $B \vDash p$ for some finite subset $B \subseteq A$ (compactness). ${ }^{10}$

See Propositions 2 and 3 for a list of useful properties under L1-L3 plus perhaps L4,L5 (a "toolkit" in general logics). In summary, the conditions L1-L3 (plus perhaps L4,L5) are sufficiently weak for representing many real-world aggregation problems, and sufficiently strong for deriving many interesting results; they are therefore a possible framework to study judgment aggregation for general logics. All this is argued more carefully later.

Note that the only logical connective that we require is negation $\neg$. For some purposes it may be useful to require in addition that the logic contains conjunction $\wedge$ (which implies that any other truth-functional connective, e.g. disjunction $\vee$ and material conditional, are expressible). One thereby gains some flexibility, and loses little generality since virtually all interesting logics contain $\neg$ and $\wedge .{ }^{11}$

\footnotetext{
${ }^{9}$ If L4 is considered too strong, different weakenings of it are imaginable. For instance, one might restrict the statement in L4 to consistent sets $A$. Also the following two conditions are (under L1-L3) weaker than L4: each inconsistent set entails every proposition (often this rather than L4 is called "non-paraconsistency"); for any $A, B \subseteq \mathbf{L}$ and $p \in \mathbf{L}$, if $A \vDash q$ for all $q \in B$, and $B \vDash p$, then $A \vDash p$ (this might be called transitivity).

${ }^{10}$ The validity of L5 can often be established as follows. First, L5 obviously holds if $\vDash$ is in fact a syntactic provability relation $\vdash$ (see Section 2.1), where every "proof" uses only finitely many of the premises. Second, L5 holds if $\vDash$ is semantic entailment, but a complete and sound proof technique is known whose proofs use only finitely many of the premises; i.e. L5 is here a corollary of a completeness and soundness theorem. However, for some important logics (e.g. those of ch. 5.6-5.7 in Priest 2001) no such theorem is currently known.

${ }^{11}$ One then has to assume that $\mathbf{L}$ contains $(p \wedge q)$ for all $p, q \in \mathbf{L}$. But this alone does not suffice, as one will also want to ensure that the "right" logical interconnections hold, e.g. that $\{p, q\} \vDash p \wedge q$, $\neg p \vDash \neg(p \wedge q)$, etc. One might do this as follows. Define $\mathbf{L}_{0}$ as the set of propositions in $\mathbf{L}$ that are not of the negated form $\neg p$ or conjunctive form $(p \wedge q)$. ( $\mathbf{L}_{0}$ contains of the "truth-functionally non-decomposable" propositions, but note that the connectives $\neg$ or $\wedge$ may occur "in" propositions $p \in \mathbf{L}_{0}$, e.g. when $p$ is $\forall x(P x \wedge \neg Q x)$.) Under the standard truth-functional rules, the truth values of the propositions in $\mathbf{L}_{0}$ should determine those of all other propositions. A evaluation of $\mathbf{L}_{0}$ is a function $f: \mathbf{L}_{0} \rightarrow\{T, F\}$, called consistent if $\left\{p \in \mathbf{L}_{0}: f(p)=T\right\} \cup\left\{\neg p: p \in \mathbf{L}_{0} \& f(p)=F\right\}$ is consistent; $f$ can be extended uniquely to a function $\bar{f}: \mathbf{L} \rightarrow\{T, F\}$ by requiring $\left.\bar{f}\right|_{\mathbf{L}_{0}}=f$ and, for all $p, q \in \mathbf{L}, f(\neg p) \neq f(p)$ and $f((p \wedge q))=T \Leftrightarrow f(p)=f(q)=T$. To enforce the standard truth-functional calculus for $\neg$ and $\wedge$, one might impose the following condition, satisfied by all logics presented in Section 3. $\mathrm{L}_{\wedge}$ : (i) for all $p, q \in \mathbf{L}$ we have $(p \wedge q) \in \mathbf{L}$; (ii) for all $A \subseteq \mathbf{L}$ and $p \in \mathbf{L}$, $A \vDash p$ if and only if [for each consistent evaluation $f$ of $\mathbf{L}_{0}$, if $\bar{f}(q)=T$ for all $q \in A$ then $\bar{f}(q)=T$ ]. $\mathrm{L}_{\wedge}$ ensures for instance, for all $p, q \in \mathbf{L}$, that $\{p, q\} \vDash p \wedge q, \neg q \vDash \neg(p \wedge q)$, and if $\{p, q\}$ is inconsistent $p \vDash p \wedge \neg q$. ( $\mathrm{L}_{\wedge}$ far from fully specify $\vDash$ : it leaves open what evaluations of $\mathbf{L}_{0}$ are consistent, which is not a matter of truth-functional calculus.) Since $L_{\wedge}$ guarantees that entailment follows the standard truth-functional rules for $\neg$ and $\wedge$, it also guarantees that (in)consistency respects these rules. On the other hand, $\mathrm{L}_{\wedge}$ might be seen as a bit strong; it for instance implies L4 (given L1-L3). If one wants to enforce the standard truth-functional rules only for (in)consistency but not for entailment, one could modify $\mathrm{L}_{\wedge}$ by replacing (ii) by the following condition: a set $A \subseteq \mathbf{L}$ is consistent if and only if there exists a consistent evaluation $f$ of $\mathbf{L}_{0}$ such that $\bar{f}(q)=T$ for all $q \in A$. Under this weakened
} 


\subsection{The basic notions of judgment aggregation}

I now define the familiar notions of judgment aggregation for a general logic $(\mathbf{L}, \vDash)$ of type L1-L3 (plus perhaps L4, L5). Consider a group of $n$ individuals denoted $1, \ldots, n$ $(n \geq 2)$, having to make collective judgments on interrelated propositions.

The agenda. The agenda (containing the propositions under consideration) is any non-empty set $X \subseteq \mathbf{L}$ that is the union of pairs $\{p, \neg p\}$ with $p$ not itself a negated proposition. The starting example has the agenda $X=\{a, \neg a, a \rightarrow b, \neg(a \rightarrow$ $b), b, \neg b\}$, where $\rightarrow$ should be a subjunctive conditional and $(\mathbf{L}, \vDash)$ a conditional logic, as explained later. By saying that $X$ contains the propositions under consideration, I am vague on purpose, in order to cover different types of aggregation problems. The standard case is surely that $X$ contains propositions of which the group has to decide whether or not to believe them (e.g. "higher interest rates reduce inflation"). ${ }^{12}$

Judgment sets. A judgment set (held by an individual or the collective) is a subset $A \subseteq X$, where $p \in A$ means " $p$ is accepted". Here, the meaning of "acceptance" depends on the type of aggregation problem, as just indicated. In the (standard) case of a decision problem about reaching beliefs, "accepting" $p$ is "believing" $p .{ }^{13} \mathrm{~A}$ judgment set $A$ is

- complete if it contains at least one member of each pair $p, \neg p \in X$;

- weakly consistent if it contains at most one member of each pair $p, \neg p \in X ;{ }^{14}$

- consistent if (see Definition 1) there is no $p \in \mathbf{L}$ with $A \vDash p$ and $A \vDash \neg p$;

- deductively closed if, for each $p \in X$, if $A \vDash p$ then $p \in A ;{ }^{15}$

- fully rational if $A$ satisfies completeness and consistency (and so all other rationality conditions by Proposition 1).

For instance, for the above example agenda $X$, the judgment set $A=\emptyset$ is consistent and deductively closed but incomplete, the judgment set $A=\{a, a \rightarrow b, \neg b\}$ is complete but only weakly consistent and not deductively closed, and the judgment set $A=\{a, \neg(a \rightarrow b), b\}$ is fully rational (since $\rightarrow$ is a subjunctive conditional, as defined below).

$\mathrm{L}_{\wedge}$, for all $p, q \in \mathbf{L}$ the set $\{p, q, \neg(p \wedge q)\}$ is still inconsistent, but $\{p, q\}$ needn't entail $p \wedge q$. $\mathrm{L}_{\wedge}$ no longer implies L4.

${ }^{12}$ One can conceive other types of decision problems: ones where $X$ contains propositions of which the question is whether to desire them (e.g. "Western products are sold all over the world"), or ones where $X$ contains propositions of which the question is whether to make them true (e.g., for the board of a central bank, "interest rates are reduced"). (It would be problematic though to mix different types of decision problems within the same agenda $X$.) I should note that a decision problem can often be transformed into one about beliefs. In particular, one about desires can be transformed by replacing each proposition $p$ by the proposition "it is desirable that $p$ ". It is philosophically controversial whether "desires" are the same as "beliefs of desirability", hence whether the transformation alters the nature of the decision problem.

${ }^{13}$ Footnote 12 starts with two alternative decision problems. In the first, accepting $p$ means wanting that $p$. In the second, for the collective accepting $p$ means making $p$ true (by action) and for an individual it means wanting that $p$ (is made true) or something else, e.g. considering it as morally good that $p$. In the jargon of philosophical logic, each notion of "acceptance" is a particular propositional attitude.

${ }^{14}$ List and Pettit (2002) call this condition "consistency".

${ }^{15}$ A subtly different definition of deductive closure is: $A \subseteq X$ is deductive closed if $A$ contains every proposition $p \in X$ entailed by some consistent subset of $A$. The difference between both definitions arises only if $A$ is inconsistent. Proposition 1 holds for both definitions. 
The various rationality conditions are interrelated as follows: ${ }^{16}$

Proposition 1 Let L1-L3 hold. For any judgment set $A \subseteq X$,

(a) consistency implies weak consistency, and the two are equivalent given deductive closure and L4;

(b) full rationality implies the conjunction of completeness, weak consistency and deductive closure, and the two are equivalent given $L 4$.

Proof. See Section 4, where we will have the tools for the proof.

For some agendas $X$, the question of whether a judgment set $A \subseteq X$ is consistent or whether it is deductively closed is non-trivial, for reasons similar to those why scientists may have a hard time showing contradictions or deriving theorems. ${ }^{17}$

Aggregation rules. A profile is an $n$-tuple $\left(A_{1}, \ldots, A_{n}\right)$ of (individual) judgment sets. A (judgment) aggregation rule is a function $F$ assigning to each profile $\left(A_{1}, \ldots, A_{n}\right)$ in a given set of admissible profiles a (collective) judgment set $F\left(A_{1}, \ldots, A_{n}\right)=A$. The set of admissible profiles is called the domain of $F$, written Domain $(F)$. All common requirements on aggregation rules (anonymity, universal domain, etc.) can easily be stated in our general framework, as they actually appeal only to very general (i.e. not logic-specific) features. For instance:

Universal Domain. The domain of $F, \operatorname{Domain}(F)$, is the set of all profiles $\left(A_{1}, \ldots\right.$, $A_{n}$ ) of fully rational judgment sets.

Collective Rationality. The collective judgment set $F\left(A_{1}, \ldots, A_{n}\right)$ is fully rational for every profile $\left(A_{1}, \ldots, A_{n}\right) \in \operatorname{Domain}(F)$.

Independence. For any proposition $p \in X$ and profiles $\left(A_{1}, \ldots, A_{n}\right),\left(A_{1}^{*}, \ldots\right.$, $\left.A_{n}^{*}\right) \in \operatorname{Domain}(F)$, if [for all individuals $i, p \in A_{i}$ if and only if $p \in A_{i}^{*}$ ] then $\left[p \in F\left(A_{1}, \ldots, A_{n}\right)\right.$ if and only if $\left.p \in F\left(A_{1}^{*}, \ldots, A_{n}^{*}\right)\right]$.

Of these requirements, the first one ensures that $F$ always produces a decision (provided individuals are fully rational), and the second one ensures that the decision is always fully rational. The third (more controversial) one is analogous to Arrow's independence of irrelevant alternatives in preference aggregation, and prescribes propositionwise aggregation/voting: the collective judgment on any given proposition $p \in X$ should be determined solely by the individual judgments on this proposition. Let me mention three aggregation rules, each defined for all profiles $\left(A_{1}, \ldots, A_{n}\right)$ in the universal domain.

- Majority rule: $F\left(A_{1}, \ldots, A_{n}\right)=\{p \in X$ : for more than half of the persons $i$, $\left.p \in A_{i}\right\}$; this rule satisfies independence, but violates collective rationality even for simple agendas, as seen in the introduction.

\footnotetext{
${ }^{16}$ In the case of classical propositional logic, similar results are shown in List (2004b).

${ }^{17}$ The "rationality" questions about judgment sets may even be undecidable in the technical sense (e.g. when $(\mathbf{L}, \vDash)$ is a sufficiently rich first-order predicate logic and $X=\mathbf{L}$ ): there exists no algorithm that determines whether or not a judgment set (input) is consistent/deductively closed. In this case also the universal domain (defined below) is an an undecidable set.
} 
- Dictatorship by person $j: F\left(A_{1}, \ldots, A_{n}\right)=A_{j}$, the dictator's judgment set; this rule satisfies both independence and collective rationality, but is undemocratic.

- Premise-based rule for the above agenda $X=\{a, \neg a, a \rightarrow b, \neg(a \rightarrow b), b, \neg b\}$ and an odd group size $n: F\left(A_{1}, \ldots, A_{n}\right)$ contains

- a premise $p \in\{a, \neg a, a \rightarrow b, \neg(a \rightarrow b)\}$ if and only if for more than half of the persons $i p \in A_{i}$ (majority voting on premises);

- a conclusion $c \in\{b, \neg b\}$ if and only if $P \vDash c$, where $P$ is the set of premises in $F\left(A_{1}, \ldots, A_{n}\right)$ (no vote taken on conclusions!).

Unlike majority rule, this rule generates consistent judgment sets, but it violates independence because of the decision method used for conclusions.

\section{Decision problems and the logics to represent them}

I now present several types of judgment aggregation problems, and define particular $\operatorname{logics}(\mathbf{L}, \models)$ that can represent them. As these logics satisfy L1-L5, the general logics model covers all these aggregation problems. The reader might be struck by the large variety of logics, and their complexity compared to the simple conditions L1-L5. The general logics model is not affected by the complexity of particular logics, as it draws only on L1-L3 (plus perhaps L4,L5). So some readers might skip the details of the definitions of particular logics.

\subsection{Decision problems with conjunctions and disjunctions: repres- entable in classical propositional logic}

Classical propositional logic (used so far in judgment aggregation) can represent decision problems involving only truth-functional connectives, such as "and", "or" and "not". ${ }^{18}$ For example, the supervisory board of a loss-making Western European company might debate the following propositions:

$a$ : A factory should be closed down.

$b:$ A new factory should be created in Eastern Europe.

$a \wedge b$ : A factory should be closed down and a new one created in Eastern Europe.

The agenda is thus $X=\{a, \neg a, b, \neg b, a \wedge b, \neg(a \wedge b)\}$ in a classical propositional $\operatorname{logic}(\mathbf{L}, \vDash)$ defined as follows.

Classical propositional logic. The language $\mathbf{L}$ is the (smallest) set such that

- $\mathbf{L}$ contains each given atomic proposition $a, b, \ldots$, and

- if $\mathbf{L}$ contains $p$ and $q$, it also contains the negation $\neg p$ ("not $p$ ") and the conjunction $(p \wedge q)$ ( " $p$ and $q$ ").

Based on $\neg$ and $\wedge$, any other truth-functional connectives can be defined; for instance, the disjunction $(p \vee q)$ ( " $p$ or $q$ ") stands for $\neg(\neg p \wedge \neg q)$ (another example is the material conditional discussed later). To simplify, for this and all following logics

\footnotetext{
${ }^{18} \mathrm{~A} k$-place connective $(k \geq 1)$ is truth-functional if the truth-value of a proposition obtained by applying the connective to $k$ propositions $p_{1}, \ldots, p_{k}$ is determined by the truth-values of $p_{1}, \ldots, p_{k}$ (e.g. the 2-place connective $\vee$ is truth-functional because the truth-value of $p \vee q$ is determined by the truth-values of $p$ and $q$ ). Thus the truth condition for a $k$-place truth-functional connective is given by a $k$-place Boolean function $B:\{T, F\}^{k} \rightarrow\{T, F\}$ (e.g. $\neg$ has the 1-place Boolean function $B:\{T, F\} \rightarrow\{T, F\}$ with $B(T)=F$ and $B(F)=T)$. In following subsections we will encounter non-truth-functional connectives.
} 
I often drop brackets when there is no ambiguity, e.g. I write $a \wedge \neg(b \vee c) \wedge d$ for $(a \wedge(\neg(b \vee c) \wedge d))$.

To define entailment $\vDash$ on $\mathbf{L}$, let an interpretation be a ("truth") function $v: \mathbf{L} \rightarrow$ $\{T, F\}$ that assigns to each proposition a truth value $T$ ("true") or $F$ ("false"), such that, for any propositions $p, q \in \mathbf{L}$,

$(\neg) v(\neg p)=T$ if and only if $v(p)=F$,

$(\wedge) v(p \wedge q)=T$ if and only if $v(p)=T$ and $v(q)=T$.

Note that $(\neg)$ and $(\wedge)$ imply the rules (truth conditions) of any other connectives defined in terms of $\neg$ and $\wedge$; for instance,

$(\vee) v(p \vee q)=T$ if and only if $v(p)=T$ or $v(q)=T$.

Each interpretation $v$ stands for "one way the world could be". Entailment is then defined as follows: a set $A \subseteq \mathbf{L}$ entails a proposition $p \in \mathbf{L}(A \vDash p)$ if for every interpretation $v$ with $v(q)=T$ for all $q \in A$ we have $v(p)=T$. Informally, $A$ entails $p$ in case $p$ is true whenever each $q \in A$ is true. For instance, $p, q \vDash p \wedge q$ (since $v(p)=v(q)=T$ implies $v(p \wedge q)=T$ by $(\wedge)$ ), $a \vDash \neg \neg a$ (since $v(a)=T$ implies $v(\neg a)=F$ by $(\neg)$, which implies $v(\neg \neg a)=T$ by $(\neg)$ ), $\neg a, a \vee b \vDash b$ (since $v(\neg a)=v(a \vee b)=T$ implies $v(a)=F$ and $[v(a)=T$ or $v(b)=T]$, hence $v(b)=T)$, etc.

Note that $A \subseteq \mathbf{L}$ is consistent (i.e. entails no pair $p, \neg p \in \mathbf{L}$ ) if and only there is an interpretation under which each $p \in A$ is true. Informally, $A$ is consistent in case its members can be simultaneously true. For instance, $\{\neg a, a \vee b\}$ is consistent (take $v(a)=F$ and $v(b)=T)$, but $\{a, \neg a\}$ is inconsistent.

\subsection{Decision problems with conditional statements: representable in conditional logics}

Now consider aggregation problems involving (bi)conditional statements $p \rightarrow q$ ("if $p$ then $q$ ") or $p \leftrightarrow q$ (" $p$ if and only if $q$ "), such as in most standard examples given in the literature. How should $\rightarrow$ and $\leftrightarrow$ be formally modelled? I will discuss three alternative routes: that of material conditionals (so far standard), that of strict conditionals, and that of fully-fleshed subjunctive conditionals.

The route taken so far in the literature uses the classical propositional logic $(\mathbf{L}, \vDash)$ defined in Section 3.1, and identifies $\rightarrow$ and $\leftrightarrow$ with the material conditional $\stackrel{\text { mat }}{\rightarrow}$ and biconditional $\stackrel{\text { mat }}{\leftrightarrow}$, defined as follows. For any $p, q \in \mathbf{L}, p \stackrel{\text { mat }}{\rightarrow} q$ stands for $\neg(p \wedge \neg q)$ ("not $[p$ and not- $q]$ "), or equivalently for $\neg p \vee q$ ("not- $p$ or $q$ "); and $p \stackrel{\text { mat }}{\leftrightarrow} q$ stands for $(p \stackrel{\text { mat }}{\rightarrow} q) \wedge(q \stackrel{\text { mat }}{\rightarrow} p)$. Thus the truth conditions for $\stackrel{\text { mat }}{\rightarrow}$ and $\stackrel{\text { mat }}{\leftrightarrow}$ are:

$(\stackrel{\text { mat }}{\rightarrow}) v(p \rightarrow q)=T$ if and only if $v(p)=F$ or $v(q)=T$;

$(\stackrel{\mathrm{mat}}{\leftrightarrow}) v(p \leftrightarrow q)=T$ if and only if $v(p)=v(q)$.

Thus $\stackrel{\text { mat }}{\rightarrow}$ validates the entailment $p, p \stackrel{\text { mat }}{\rightarrow} q \vDash q$ ("modus ponens"), as well as $\neg p \vDash p \stackrel{\text { mat }}{\rightarrow} q$ and $q \vDash p \stackrel{\text { mat }}{\rightarrow} q$ (the two "paradoxes of the material conditional").

Material conditionals raise well-known problems and misrepresent the intended meaning of most conditional statements in normal language (e.g. Priest 2001). The statement "if it's the 15th century then people drive cars" is true as a material conditional because it's not the 15th century (and also because people drive cars). This clash between our intuition and the material conditional is due to the fact that, usually, "if $a$ then $b$ " does not say something about the actual truth values of $a$ and $b$, but something about $b$ 's truth value in other (perhaps non-actual) worlds in which $a$ 
holds, e.g. worlds where it's the 15th century. The statement "if it's the 15th century then people drive cars" does not mean "either it's not the 15th century or we drive cars" (true), but it means "in a world of the 15th century, people drive cars" (false).

Also in judgment aggregation, the relevant conditionals are usually not material, as argued in Dietrich (2005). For instance, the conditional

$a \rightarrow b$ : "if the birth rate is too low then more immigration is needed"

does not have the material meaning, as it does not mean

$\neg a \vee b$ : "the birth rate is not too low or more immigration is needed".

Rather it means "in the case (the world(s)) where the birth rate is too low, more immigration is needed". Under the latter reading, it is perfectly consistent to reject $a$ without accepting $a \rightarrow b$; but if $\rightarrow$ were $\stackrel{\text { mat }}{\rightarrow}$ then $\neg a$ would entail $a \rightarrow b$ by $(\stackrel{\text { mat }}{\rightarrow})$.

To represent conditionals more adequately, I now define two logics $(\mathbf{L}, \vDash)$ based on possible-worlds semantics, which goes back to Kripke (1963) and others and is now widely used; for reference, e.g. Priest (2001).

In the first logic, $S 5$, the conditional "if $p$ then $q$ " is declared true if $q$ holds in every world in which $p$ holds. To express "in every world", the language $\mathbf{L}$ will contain a necessity operator $\square$, in terms of which conditionals will be defined.

The modal logic $S 5$. The language $\mathbf{L}$ is the (smallest) set such that

- $\mathbf{L}$ contains each given atomic proposition $a, b, \ldots$, and

- if $\mathbf{L}$ contains $p$ and $q$, it also contains $\neg p,(p \wedge q)$, and $\square p$ ("necessarily $p$ ").

Like in classical propositional logic, any truth-functional operators $(\vee, \stackrel{\text { mat }}{\rightarrow}, \stackrel{\text { mat }}{\leftrightarrow}$ etc. $)$ can be defined using $\neg, \wedge$. Other non-truth-functional operators like the strict (or strong) conditional $\stackrel{\text { str }}{\rightarrow}$ and biconditional $\stackrel{\text { str }}{\rightarrow}$ can be defined using $\square$ : for any $p, q \in \mathbf{L}$, $p \stackrel{\text { str }}{\longrightarrow} q$ stands for $\square(p \stackrel{\text { mat }}{\rightarrow} q)$, or equivalently for $\square(\neg p \vee q)$ ("necessarily, not- $p$ or $q$ "); and $p \stackrel{\text { str }}{\rightarrow} q$ stands for $(p \stackrel{\text { str }}{\rightarrow} q) \wedge(q \stackrel{\text { str }}{\rightarrow} p)$.

While for classical propositional logic an interpretation is given by a single truth function, an S5-interpretation is a pair $\left(W,\left(v_{w}\right)_{w \in W}\right)$, where:

- $W$ is a non-empty set of objects called (possible) worlds;

- $\left(v_{w}\right)_{w \in W}$ is a family of ("truth") functions $v_{w}: \mathbf{L} \rightarrow\{T, F\}$, assigning to each proposition $p \in \mathbf{L}$ its truth value $v_{w}(p)$ in world $w \in W$, such that, for all worlds $w \in W$ and propositions $p, q \in \mathbf{L}$, the truth function $v=v_{w}$ satisfies $(\neg),(\wedge)$, and

(口) $v(\square p)=T$ if and only if $v_{w^{\prime}}(p)=T$ for all world $w^{\prime} \in W$.

From $(\square)$ we can immediately deduce the truth conditions for $\stackrel{\text { str }}{\longrightarrow}$ and $\stackrel{\text { str }}{\leftrightarrow}$ :

$(\stackrel{\text { str }}{\rightarrow}) v_{w}(p \stackrel{\text { str }}{\longrightarrow} q)=T$ if and only if $v_{w^{\prime}}(q)=T$ for all worlds $w^{\prime} \in W$ with $v_{w^{\prime}}(p)=T$

$(\stackrel{\text { str }}{\leftrightarrow}) v_{w}(p \stackrel{\text { str }}{\leftrightarrow} q)=T$ if and only if $v_{w^{\prime}}(p)=v_{w^{\prime}}(q)$ for all worlds $w^{\prime} \in W$.

So the strict conditionals $p \stackrel{\text { str }}{\rightarrow} q$ holds in a world just in case $q$ holds in every world in which $p$ holds. The truth value of $p \stackrel{\text { str }}{\rightarrow} q$ depends not just on the actual world: the operator $\stackrel{\operatorname{str}}{\rightarrow}$ is not truth-functional. Interpretations $\left(W,\left(v_{w}\right)_{w \in W}\right)$ differ in what worlds are considered possible, and in what propositions are true in them. Presumably, in our immigration example the set of possible worlds $W$ include worlds with a bright economic future, in which $b$ is true, and worlds where just the opposite holds. As another example, if the only relevant aspect is the season, we may put $W=\{S p, S u, A u, W i\}$, where the atomic proposition "European trees are loosing their leaves" is true in the world $A u$ and false in all world $w \in W \backslash\{A u\}$. 
By definition, a set $A \subseteq \mathbf{L}$ entails a proposition $p \in \mathbf{L}(A \vDash p)$ if for every $S 5$ interpretation $\left(W,\left(v_{w}\right)_{w \in W}\right)$ and every world $w \in W$ with $v_{w}(q)=T$ for all $q \in A$ we have $v_{w}(p)=T$. Informally, $A$ entails $p$ if, whenever each $q \in A$ is true, $p$ is true. The strict conditional still satisfies modus ponens as $a, a \stackrel{\text { str }}{\longrightarrow} b \vDash b$. But it avoids the two paradoxes of the material conditional as $\neg a \not \models a \stackrel{\text { str }}{\rightarrow} b$ and $b \not \models a \stackrel{\text { str }}{\longrightarrow} b$. This is the main advantage of $\stackrel{\text { str }}{\longrightarrow}$ over $\stackrel{\text { mat }}{\rightarrow}$. Note also that $A$ is consistent (i.e. entails no pair $p, \neg p)$ if and only if some world of some $S 5$-interpretation makes all $q \in A$ true.

In judgment aggregation, it makes a considerable difference whether strict or material conditionals are used. To illustrate this, consider again our immigration example with agenda $X=\{a, \neg a, a \rightarrow b, \neg(a \rightarrow b), b, \neg b\}$, and let us see which judgment sets $A \subseteq X$ are declared fully rational (i.e. consistent and complete) if

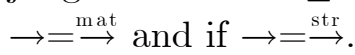

- If $\rightarrow=\stackrel{\text { mat }}{\rightarrow}$, the only fully rational judgment sets are $\{a, a \rightarrow b, b\},\{\neg a, a \rightarrow b, b\}$, $\{\neg a, a \rightarrow b, \neg b\},\{a, \neg(a \rightarrow b), \neg b\}$.

- If $\rightarrow=\stackrel{\text { str }}{\rightarrow}$, there are three additional fully rational judgment sets, namely $\{a, \neg(a$ $\rightarrow b), b\},\{\neg a, \neg(a \rightarrow b), b\},\{\neg a, \neg(a \rightarrow b), \neg b\}$. A negated strict conditional $\neg(a \rightarrow b)$ is consistent with any truth values of $a$ and $b$ !

Nevertheless, strict conditionals - historically the first attempt to formalise nonmaterial conditionals - face other, more subtle, problems, which suggest that they do still not fully faithfully represent the intended meaning of many conditional statements. Most problems of strict conditionals remain if we move to more refined modal logics than $S 5$, i.e. to modal logics of type $K_{\Delta}$ discussed later. Often, only subjunctive conditionals are considered fully adequate. For, "if $a$ then $b$ " often means not that $b$ holds in every world where $a$ holds (strict conditional), but that $b$ holds in worlds similar to the actual world except that $a$ is true (subjunctive conditional). Thus the meaning of "if $a$ then $b$ " is often: if $a$ were true - if the actual world were modified so that $a$ becomes true ceteris paribus - then $b$ would be true. One might even interpret "if $a$ then $b$ " as "in the closest world(s) in which $a$ holds, $b$ holds".

Subjunctive conditionals were formalised by Stalnaker (1968) and D. Lewis (1973) using conditional logics and have become well-established. Let me introduce $C^{+}$, a standard version of conditional logic.

The conditional logic $C^{+}$. The language $\mathbf{L}$ is the (smallest) set such that

- $\mathbf{L}$ contains each given atomic proposition $a, b, \ldots$, and

- if $\mathbf{L}$ contains $p$ and $q$, then $\mathbf{L}$ also contains $\neg p,(p \wedge q)$, and $p \stackrel{\text { sub }}{\longrightarrow} q$ ("if $p$ then $q$ " in the subjunctive sense of "if $p$ were true then $q$ would be true").

Again, any truth-functional operators $(\vee, \stackrel{\text { mat }}{\rightarrow}, \stackrel{\text { mat }}{\leftrightarrow}$ etc. $)$ can be defined in terms of $\neg, \wedge$. Moreover, the subjunctive biconditional $\stackrel{\text { sub }}{\leftrightarrow}$ is definable using $\stackrel{\text { sub }}{\rightarrow}$ : for any $p, q \in \mathbf{L}, p \stackrel{\text { sub }}{\leftrightarrow} q$ stands for $(p \stackrel{\text { sub }}{\rightarrow} q) \wedge(q \stackrel{\text { sub }}{\rightarrow} p)$.

To define $\vDash$, first let a $C^{+}$-interpretation be a triple $\left(W,\left(R_{p}\right)_{p \in \mathbf{L}},\left(v_{w}\right)_{w \in W}\right)$, where:

- $W$ is again a non-empty set of (possible) worlds;

- $\left(R_{p}\right)_{p \in \mathbf{L}}$ is a family of binary relation on $W\left(w R_{p} w^{\prime}\right.$ means "world $w^{\prime}$ is similar to world $w$, and $p$ is true in $\left.w^{\prime \prime \prime}\right)$, such that, for any $w, w^{\prime} \in W$ and $p \in \mathbf{L}$,

- if $w R_{p} w^{\prime}$ then $v_{w^{\prime}}(p)=T$ (an obvious requirement given what $w R_{p} w^{\prime}$ means), and

- if $v_{w}(p)=T$ then $w R_{p} w$ (since $w$ is similar to itself); 
- $\left(v_{w}\right)_{w \in W}$ is a family of ("truth") functions $v_{w}: \mathbf{L} \rightarrow\{T, F\}$, assigning to each proposition $p \in \mathbf{L}$ a truth value $v_{w}(p)$ in world $w \in W$, such that, for all $w \in W$ and $p, q \in \mathbf{L}$, the truth function $v=v_{w}$ satisfies $(\neg),(\wedge)$, and

$(\stackrel{\text { sub }}{\longrightarrow}) v_{w}(p \stackrel{\text { sub }}{\rightarrow} q)=T$ if and only if $v_{w^{\prime}}(q)=T$ for all worlds $w^{\prime} \in W$ with $w R_{p} w^{\prime}$.

So $p \stackrel{\text { sub }}{\longrightarrow} q$ is true in world $w$ just in case $q$ is true in every world $w^{\prime}$ similar to $w$ and with true $p$. This captures the above intuition for subjunctive as opposed to strict conditionals. For instance, "if the G8 countries were not industrialised then the ozone layer would be in good order" is (probably) true as a subjunctive conditional: were the G8 countries not industrialised ceteris paribus, much of present $\mathrm{CO} 2$ emissions wouldn't occur. But it is false as a strict conditional: think of worlds in which the G8 countries are not industrialised but many other countries are.

Entailment is defined as for $S 5$, but now relative to $C^{+}$-interpretations: a set $A \subseteq \mathbf{L}$ entails a proposition $p \in \mathbf{L}(A \vDash p)$ just in case for every $C^{+}$-interpretation and every world $w$ if each $q \in A$ is true in $w$ then $p$ is true in $w$. Again, this implies that $A$ is consistent if and only if for some world of some $C^{+}$-interpretation each $q \in A$ is true.

Like the strict conditional $\stackrel{\text { str }}{\longrightarrow}$ in $S 5, \stackrel{\text { sub }}{\longrightarrow}$ satisfies modus ponens (as $p, p \stackrel{\text { sub }}{\longrightarrow} q \vDash q$ ) but is not vulnerable to the paradoxes of the material conditional (as $\neg a \not \models a \stackrel{\text { sub }}{\longrightarrow} b$ and $b \not \models a \stackrel{\text { sub }}{\longrightarrow} b$ ). In judgment aggregation, modelling $\rightarrow$ as $\stackrel{\text { str }}{\longrightarrow}$ or as $\stackrel{\text { sub }}{\longrightarrow}$ often makes little difference in the sense that we obtain the same fully rational judgment sets for many agendas, including for the above agenda $X=\{a, \neg a, a \rightarrow b, \neg(a \rightarrow b), b, \neg b\}$. But sometimes differences arise; for instance, $\stackrel{\text { str }}{\rightarrow}$ is transitive (i.e. $p \stackrel{\text { str }}{\rightarrow} q, q \stackrel{\text { str }}{\rightarrow} r \vDash p \stackrel{\text { str }}{\rightarrow} r$ for all $p, q, r \in \mathbf{L})$ but $\stackrel{\text { sub }}{\longrightarrow}$ isn't.

\subsection{Decision problems with modal statements: representable in modal logics}

Modal operators are used to represent phrases in front of propositions such as "it is desirable that", "it is ethically required that", "it is in our interest that", "it is feasible that", "it is probable that", "it is known that" etc. A modal operator in front of $p$ does not say that $p$ is true or false, but that $p$ is desirable, or morally required, etc. There are various ways in which modal operators can be relevant in judgment aggregation. Let me give two examples.

Non-separable decisions on acts. Many decision problems consist in deciding on collective acts. Consider act-describing propositions, such as "income taxes are raised", "indirect taxes are raised", "the budget deficit is reduced". Some acts may be non-separable from others: whether the former should be taken depends on whether the latter are taken. To represent this, one may use a modal operator $S$ standing for "it is desirable that", and consider an agenda $X$ that contains the following two types of propositions (and their negations):

- propositions of the form $S(p)$, where $p$ is a (perhaps negated) act-describing proposition; $S(p)$ could be "it is desirable that income taxes are raised", in short "income taxes should be raised";

- conditional statements of the form $p \rightarrow S(q)$, where $p$ and $q$ are (perhaps negated) act-describing propositions; $p \rightarrow S(q)$ could be "if the budget deficit is not reduced, then income taxes should not be raised". 
A judgment set $A \subseteq X$ then states that certain acts should (not) be taken, unconditionally or conditionally on other acts being (not) taken. Note that this approach differs from the way preference aggregation handles non-separability.

Probabilistic statements. In a private communication, Ruwin Gekker drew my attention towards the importance of probabilistic statements of the form "it is probable that $p$ " (in short: "probably $p$ "), where $p$ is a factual proposition such as "carbon dioxide emissions are a cause of global warming" or "global warming will continue over the next 10 years". It may be interesting to use a modal operator $P$ for "probably" and to consider an agenda $X$ containing:

- propositions of the form $P(p)$, where $p$ is a factual proposition; $P(p)$ could be "Probably carbon dioxide emissions are a cause of global warming";

- conditional statements of the form $p \rightarrow P(q)$, where $p$ and $q$ are factual propositions; $p \rightarrow P(q)$ could be "if carbon dioxide emissions are a cause of global warming then probably global warming will continue over the next 10 years".

A judgment set $A \subseteq X$ expresses probabilistic beliefs about the world. These probabilistic beliefs are less exact than those expressed in a probability function (how probable is "probably"?); however, it is more realistic that a person can submit a judgment set $A \subseteq X$ than a full probability function.

Each type of modal operator requires its own semantics, and hence its own logic $(\mathbf{L}, \vDash)$. For instance, it may be desirable that the above operator $S$ satisfies $S(p), S(q) \vDash S(p \wedge q)$ for all $p, q \in \mathbf{L}$ (if $p$ and $q$ are desirable, so is $p \wedge q$ ), but surely not that the above operator $P$ satisfies $P(p), P(q) \vDash P(p \wedge q)$ for all $p, q \in \mathbf{L}$ (if $p$ and $q$ are each probable, $p \wedge q$ need not be probable). While there are many modal logics, most of them satisfy the conditions L1-L5 of the present model of judgment aggregation. Many of these logics share a common feature: they are based on possible-worlds semantics, like the formalisation of conditional statements discussed in Section 3.2. The reason is that a modal operator in front of a proposition $p$ can often be interpreted as stating that $p$ holds in every (or in some) possible world, under an appropriate notion of possibility: "it is desirable that $p$ " means " $p$ holds in every world respecting our desires"; "it is ethically required that $p$ " means " $p$ holds in every (morally) permissible world"; "it is in our interest that $p$ " means " $p$ holds in every world respecting our interests", etc. But there are also exceptions where possible worlds semantics are inappropriate, notably regarding the operator "it is probable that"; see for instance Gekker 2003.

Some decision problems require the use of a multi-modal logic (which contains more than one modal operator), or a logic with a modal operator and a subjunctive conditional $\rightarrow$, as might be the case in the two examples above. ${ }^{19}$

In a modal logic $(\mathbf{L}, \vDash)$ with a (single) modal operator $\square, \mathbf{L}$ is defined just like in the modal logic $S 5$ (see Section 3.2), and $\square p$ means that $p$ holds necessarily according to some type of necessity such as those mentioned above. If the logic is possible-world based, holding necessarily means holding in every possible world, where a world is possible if it respects desires, or interests, or morality, or budget constraints, or is

\footnotetext{
${ }^{19}$ The adequate representation of conditional commitments, like those in our first example, is a controversial issue in deontic logic (e.g. Hintikka 1971 and Wagner Decew 1981). In particular, it is controversial whether and when $p \rightarrow S(q)$ or $S(p \rightarrow q)$ is preferrable to represent a conditional commitment. It is often argued that " $\rightarrow$ " should not be a material conditional.
} 
compatible with our information, etc. To the necessity operator $\square$ corresponds a possibility operator $\diamond$, which is definable in terms of $\square$ : for any $p \in \mathbf{L}, \diamond p$ ("it is possible that $p$ ") stands for $\neg \square \neg p$ ("it is not necessary false that $p$ "). The meaning of $\diamond$ depends on that of $\square$ : if $\square$ represents the morally necessary $\diamond$ represents the morally permissible, if $\square$ represents the financially necessary, $\diamond$ represents the affordable, etc.

How should entailment $\vDash$ on $\mathbf{L}$ be defined? Surely, the simplest possible-worlds semantics, appropriate only for some modal operators, is that of the logic $S 5$ defined in Section 3.2. In $S 5$ we for instance have, for each $p \in \mathbf{L}, \square p \vDash p$ (since if $p$ is true in every world, it is true in the actual world) and $\square p \vDash \diamond p$ (since if $p$ is true in every world it is true in some world).

But $S 5$ is far from representing all forms of modality adequately. If $\square$ stands for "it is desirable that", the entailment $\square p \vDash p$ is problematic: "it is desirable that all humans live in harmony" does not entail "all humans live in harmony". If $\square$ represents moral necessity, the entailment $\square p \vDash p$ is also problematic : being ethically required does not entail being true. What causes the problem is that $S 5$ interprets $\square p$ as meaning that $p$ holds in all worlds, rather than in certain worlds.

This suggests that one needs a notion of relative possibility: relative to a given world $w$, only certain worlds should be possible. Writing $w R w^{\prime}$ if $w^{\prime}$ is a possible world relative to world $w$, we can then define $\square p$ to hold in a world $w$ in case $p$ holds in every world $w^{\prime}$ with $w R w^{\prime}$, i.e. in every possible world if we are in $w$. $R$ establishes a binary relation on $W$.

Note that this move resembles the move done in the last subsection to construct the conditional $\operatorname{logic} C^{+}$. There we needed not one relation $R$ of "relative possibility", but for each $p \in \mathbf{L}$ a relation $R_{p}$ of "similarity subject to $p$ being true". While the relations $R_{p}$ was used to define subjunctive conditionals, which overcome problems of the strict conditional $p \stackrel{\text { str }}{\rightarrow} q(=\square(p \stackrel{\text { mat }}{\rightarrow} q))$, the present relation $R$ will be used to fix problems that modal statements $\square p$ have in $S 5$.

Of course, not any binary relation $R$ on $W$ can represent relative possibility: $R$ has to satisfy certain conditions, where these conditions crucially depend on the type of modality to be represented. Often a subset of the following conditions is appropriate: reflexivity ( $w R w$ for all $w \in W$ ), symmetry (if $w R w^{\prime}$ then $w^{\prime} R w$, for all $w, w^{\prime} \in W$ ), transitivity (if $w R w^{\prime}$ and $w^{\prime} R w^{\prime \prime}$ then $w R w^{\prime \prime}$, for all $w, w^{\prime}, w^{\prime \prime} \in W$ ), and extensibility (for all $w \in W$ there is a $w^{\prime} \in W$ such that $w R w^{\prime}$ ). For each set $\Delta$ of conditions on $R$, a corresponding modal logic can be defined; I denote it $K_{\Delta}$ after Kripke (1963), one of the founders of possible-words semantics. As the reader will notice, $S 5$ is the special case of $K_{\Delta}$ in which $\Delta$ contains a single (very strong) condition on $R$ : every world accesses every world.

The modal logic $K_{\Delta}$. The language $\mathbf{L}$ is defined as in $S 5$. Again, using $\wedge$ and $\neg$ we can define all truth-functional operators $(\vee \stackrel{\text { mat }}{\rightarrow}, \stackrel{\text { mat }}{\leftrightarrow}$, etc. $)$, and, also using $\square$, we can define non-truth-functional operators $(\stackrel{\text { str }}{\longrightarrow}, \stackrel{\text { str }}{\leftrightarrow}, \diamond$, etc. $)$.

To define entailment $\vDash$, let a $K_{\Delta}$-interpretation be a triple $\left(W, R,\left(v_{w}\right)_{w \in W}\right)$, where

- $W$ is a non-empty set of (possible) worlds;

- $R$ is a binary relation on $W$ satisfying the conditions in $\Delta ; R$ is the relative possibility relation or accessibility relation, and $w R_{p} w^{\prime}$ means "in world $w, w^{\prime}$ is possible";

- $\left(v_{w}\right)_{w \in W}$ is a family of ("truth") functions $v_{w}: \mathbf{L} \rightarrow\{T, F\}$, assigning to each 
proposition $p \in \mathbf{L}$ its truth value $v_{w}(p)$ in world $w \in W$, such that, for any $w \in W$ and $p, q \in \mathbf{L}$, the truth function $v=v_{w}$ satisfies $(\neg),(\wedge)$, and

(口) $v(\square p)=T$ if and only if $v_{w^{\prime}}(p)=T$ for all worlds $w^{\prime} \in W$ with $w R w^{\prime}$.

By $(\square)$ and the definition of $\diamond$, the truth condition for possibility is:

$(\diamond) v_{w}(\diamond p)=T$ if and only if $v_{w^{\prime}}(p)=T$ for some world $w^{\prime} \in W$ with $w R w^{\prime}$.

So $\square p(\diamond p)$ is true if $p$ is true in each (some) world that is possible relative to the actual world.

By definition, a set $A \subseteq \mathbf{L}$ entails a proposition $p \in \mathbf{L}(A \vDash p)$ if, for every world $w$ of any $K_{\Delta}$-interpretation, if each $q \in A$ is true in $w$ then $p$ is true in $w$. Again, it follows that $A$ is consistent if and only if some world of some $K_{\Delta}$-interpretation makes all $q \in A$ true.

Different sets $\Delta$ of conditions on the relative possibility relation $R$ generate different properties of entailment $\vDash$. For instance, the characteristic property of the reflexivity condition is that $\square p \vDash p$ for all $p \in \mathbf{L}$ (since if $p$ holds in every possible world then, as the actual world is possible, $p$ holds in the actual world). The characteristic property of the extensibility condition is that $\square p \vDash \diamond p$, i.e. if $p$ is necessary then $p$ is possible (since if $p$ holds in all possible worlds then, as there exists a possible world, it holds in some possible world).

It is debatable which set of conditions $\Delta$ is appropriate for the different modal operators. If $\square$ represents "it is known that" (i.e. "the available information implies that"), $\Delta$ should contain the reflexivity condition since we want $\square p \vDash p$ (if $p$ is known to be true then $p$ must be true). If $\square$ represents desirability or moral necessity then $\Delta$ should not contain the reflexivity condition since otherwise we obtain $\square p \vDash p$, a problematic entailment as noted above. It is often argued (see Priest 2001) that in order to represent moral necessity $\Delta$ should consist only of the extensibility condition (which implies $\square p \vDash \diamond p$ ).

\subsection{Preference aggregation problems: representable in a predicate logic}

Like in preference aggregation, suppose a group has to establish a collective preference relation $\succeq$ over a set of alternatives $C=\left\{c_{1}, c_{2}, \ldots, c_{k}\right\}(k \geq 2)$ based on individual preference relations. I now present two ways to model this decision problem as an instance of judgment aggregation in general logics. Following List and Pettit (2004), I will represent preferences and rationality conditions as propositions of predicate calculus.

We consider the predicate language given by the set of constants $C=\left\{c_{1}, \ldots, c_{k}\right\}$, the set of variables $V=\left\{v_{1}, v_{2}, \ldots\right\}$, the two binary predicates $R$ ("is at least as good as") and = ("is equal to"), and the standard operators $\neg$ ("not"), $\wedge$ ("and"), and $\forall$ ("for all"). Formally, the atomic propositions are the expressions $x R y$ and $x=y$, where $x, y \in C \cup V$, and the set of all propositions, $\mathbf{L}$, is defined as the (smallest) set such that

- $\mathbf{L}$ contains each atomic proposition, and

- if $\mathbf{L}$ contains $p$ and $q$, it also contains $\neg p,(p \wedge q)$, and $(\forall v) p$ for each variable $v \in V$ (let $\vee$ and $\rightarrow$ be disjunction and material conditional, defined in terms of $\wedge, \neg)$.

Then: 
(i) Each preference relation $\succeq$ on $C$ can be represented as a set of propositions

$$
A_{\succeq}:=\left\{c R c^{\prime}: c, c^{\prime} \in C \text { and } c \succeq c^{\prime}\right\} \cup\left\{\neg c R c^{\prime}: c, c^{\prime} \in C \text { and } c \nsucceq c^{\prime}\right\} \subseteq \mathbf{L},
$$

reflecting all pairwise rankings under $\succeq$.

(ii) Any rationality condition on preferences can be expressed as a proposition in $\mathbf{L}$; for instance, the completeness condition is $\forall v_{1} \forall v_{2}\left(v_{1} R v_{2} \vee v_{2} R v_{1}\right)$, and the transitivity condition is $\forall v_{1} \forall v_{2} \forall v_{3}\left(\left(v_{1} R v_{2} \wedge v_{2} R v_{3}\right) \rightarrow v_{1} R v_{3}\right)$. Let $\mathcal{R} \subseteq \mathbf{L}$ be a set, interpreted as the set of desirable rationality conditions, e.g. those of a weak order, or those of a linear order, or those of an acyclic and reflexive partial order.

(iii) The exclusiveness of the options can be expressed by the proposition $\wedge_{1 \leq j<j^{\prime} \leq k}$ $\neg\left(c_{j}=c_{j^{\prime}}\right)$ (stating that $c_{1}, \ldots, c_{k}$ are pairwise distinct), and the exhaustiveness of the options can be expressed by the proposition $\forall v_{1}\left(\vee_{1 \leq j \leq k} v_{1}=c_{j}\right)$ (stating that $c_{1}, \ldots, c_{k}$ are the only options); here, " $\wedge_{1 \leq j<j^{\prime} \leq k}$ " and " $\vee_{1 \leq j \leq k}$ " are shorthands, e.g. " $\vee_{1 \leq j \leq k} v_{1}=c_{j}$ " means $v_{1}=c_{1} \vee \ldots \vee v_{1}=c_{k}$ (where $\vee$ is defined in terms of $\wedge$ and $\neg$, as done earlier). Let $\mathcal{E}$ be the set of these two conditions.

Exogenous rationality conditions. Assume first that, as in standard preference aggregation, the rationality conditions in $\mathcal{R}$ are exogenously imposed, i.e. not subject to a decision. Then the agenda should be defined as the set $X:=X_{C}:=$ $\left\{c R c^{\prime}, \neg c R c^{\prime}: c, c^{\prime} \in C\right\}$. To make the rationality conditions and the exclusiveness and exhaustiveness conditions true by definition, let me turn them into axioms of the logic. Specifically, I consider the set of axioms $\mathcal{A}=\mathcal{R} \cup \mathcal{E}$, containing the rationality, exclusiveness and exhaustiveness conditions, and I define a preference logic by the language $\mathbf{L}$ together with the entailment relation given by:

$$
A \vDash p \text { if and only if } \begin{aligned}
& A \cup \mathcal{A} \text { entails } p \text { in the standard } \\
& \text { sense of predicate logic. }{ }^{20}
\end{aligned}
$$

In this logic, a set $A \subseteq \mathbf{L}$ is consistent (i.e. entails no pair $p, \neg p \in \mathbf{L}$ ) if and only if $A \cup \mathcal{A}$ is consistent in the standard sense of predicate logic. By consequence, the preferencetheoretic notion of rationality translates into the logical notion of consistency:

- A preference relation $\succeq$ on $C$ satisfies all rationality conditions in $\mathcal{R}$ if and only if the corresponding judgment set $A_{\succeq}$ (defined above) is consistent.

Hence, the preference aggregation problem of finding rational collective preferences becomes the judgment aggregation problem of finding consistent collective judgments.

Endogenous rationality conditions. Judgment aggregation also allows one to study a new decision problem, in which the (amount and type of) collective rationality is itself subject to a decision. To this end, let us augment the agenda by the rationality conditions, i.e. let us consider the agenda $X:=X_{C, \mathcal{R}}=\left\{c R c^{\prime}, \neg c R c^{\prime}: c, c^{\prime} \in\right.$ $C\} \cup\{r, \neg r: r \in \mathcal{R}\}$. Rather than building the rationality conditions as axioms into

\footnotetext{
${ }^{20}$ Entailment in predicate logic is defined in most standard logic textbooks, e.g. Mendelssohn (1979). I adopt here a frequent convention on the notion of an "interpretation" for predicate logics: the equality symbol $=$ (if present in the language) is by definition interpreted as the equality relation (rather than as any arbitrary binary relation). This implies that, for any constant symbols $c, c^{\prime}$, $c=c^{\prime}$ is true under an interpretation if and only if the interpretation maps $c$ and $c^{\prime}$ to the same object.
} 
the logic, consider the smaller set of axioms $\mathcal{A}:=\mathcal{E}$, containing only the exclusiveness and exhaustiveness conditions, and let the logic be the language $\mathbf{L}$ endowed with the entailment relation $\vDash$ defined by (1) using the new set of axioms $\mathcal{A}$. ${ }^{21}$

\subsection{Exogenous constraints: representable by axioms}

Decision tasks that must obey certain (exogenous) constraints can often be represented by building the constraints as axioms into the logic; an example was given in the previous subsection, where constraints like "transitivity" were built into the logic. This move turns the respectance of constraints into a matter of logical consistency of collective judgment sets. Formally, if both the propositions under decision and the constraints are expressible in some logic $(\mathbf{L}, \tilde{F})$ (e.g. the conditional logic $C^{+}$, a version of modal logic $K_{\Delta}$, or some predicate logic), then we can use a modified logic $(\mathbf{L}, \vDash)$ with entailment $\vDash$ thus defined: for any $A \subseteq \mathbf{L}$ and $p \in \mathbf{L}, A \vDash p: \Leftrightarrow A \cup \mathcal{A} \widetilde{F} p$, where $\mathcal{A} \subseteq \mathbf{L}$ is the set of constraints.

Some constraints in $\mathcal{A}$ may simply be analytic truths, such as the proposition $3>2$ and the proposition $\left(\wedge_{k \in K} p_{k}\right) \rightarrow p$, where $K$ is the set of items of a budget, $p$ is "total spending increases" and $p_{k}$ is "spending on item $k$ increases". But $\mathcal{A}$ will often also contain contingent constraints, which the group faces due to the world in which it lives; examples are (propositions expressing) legal constraints or budget constraints. For instance, the decision problem faced by the court in the classical doctrinal paradox (Kornhauser and Sager 1986) can be modelled as follows. The agenda is $X=\{a, \neg a, b, \neg b, c, \neg c\}$, where $a$ is "the defendant has broken contract $\mathrm{x}$ ", $b$ is "contract $\mathrm{x}$ is valid" and $c$ is "the defendant is guilty". Given that legal doctrine declares $c$ to hold if and only if $a$ and $b$ hold, the agenda belongs to the logic $(\mathbf{L}, \models)$ obtained by building into, say, the conditional logic $C^{+}$the axiom $c \stackrel{\text { sub }}{\leftrightarrow}(a \wedge b)$. That is, $\mathbf{L}$ is the language of $C^{+}$, and $A \vDash p$ if and only if $A \cup\{c \stackrel{\text { sub }}{\leftrightarrow}(a \wedge b)\}$ entails $p$ in $C^{+}$. In this logic, the judgment set $\{a, b, \neg c\}$ is inconsistent, as desired. If, however, the proposition $c \stackrel{\text { sub }}{\leftrightarrow}(a \wedge b)$ were not exogenously imposed but subject to a decision, the agenda would also include $c \stackrel{\text { sub }}{\leftrightarrow}(a \wedge b)$ and $\neg(c \stackrel{\text { sub }}{\leftrightarrow}(a \wedge b))$, and the logic would be simply $C^{+}$.

\subsection{Decision problems with vague propositions: representable in fuzzy logics}

Arguably, in some decision problems propositions are "vague" and hence can have truth values between "true" and "false". This might be so for "the economy is in good shape", as "in good shape" is not precisely defined. To account for vagueness, one might use a fuzzy logic $(\mathbf{L}, \vDash)$. Simple fuzzy logics $(\mathbf{L}, \vDash)$ with only truth-functional

\footnotetext{
${ }^{21}$ There is an additional subtlety. Intuitively, it is perfectly consistent to reject, say, the completeness condition while holding a complete preference relation $\succeq$ by pure coincidence. Indeed, rejecting the completeness condition is not claiming that preferences must be incomplete, but that they may be incomplete. However, assume a person holds a complete preference relation $\succeq$. His or her judgment set $A \subseteq X_{C, \mathcal{R}}$ is a superset of $A_{\succeq}$, the set of ranking judgments corresponding to $\succeq$. As one easily checks, $A_{\succeq}$ entails the completeness condition (i.e. $A_{\succeq} \vDash \forall v_{1} \forall v_{2}\left(v_{1} R v_{2} \vee v_{2} R v_{1}\right)$ ), and hence so does $A$. So (if $\bar{A}$ is deductively closed) $A$ must contain the completeness condition! Similar remarks apply to all rationality conditions. To make it possible to reject rationality conditions even if they happen to be satisfied by the actual preferences $\succeq$, one could add a modal necessity operator $\square$ in front of each rationality condition - which requires using a modal predicate logic instead.
} 
connectives can be obtained by "fuzzifying" classical propositional logic. Specifically, let the language $\mathbf{L}$ be the same as in classical propositional logic with connectives $\neg, \wedge$ (see Section 3.1), but let us redefine interpretations (hence $\vDash$ ). If we use a whole interval $[0,1]$ of truth values (the extremes 0 and 1 correspond to $F$ and $T$ in binary logics), a (fuzzy) interpretation of $\mathbf{L}$ is a function $v: \mathbf{L} \rightarrow[0,1](v(p)=\alpha$ means $p$ is true to the degree $\alpha$ ) such that, for all $p, q \in \mathbf{L}, v(\neg p)=1-v(p)$ and $v(p \wedge q)=\min \{v(p), v(q)\}$. Based on this notion of interpretations, there are different ways to define entailment $\vDash$ (e.g. Priest 2001, ch. 11). Importantly, some may violate L4 (be paraconsistent), or even violate L3. As long as L1-L3 hold, standard judgment aggregation results apply. This is so for the following definition of $\vDash$. Fix a threshold $\delta \in(1 / 2,1]$ for "sufficiently true", and let $A \vDash p$ mean that, for all interpretations $v$, if $v(q) \geq \delta$ for all $q \in A$ then $v(p) \geq \delta$.

\section{A toolkit for working with general logics}

Having considered many particular logics representing particular decision problems, we are back to full generality. I now state what I take to be the basic technical tools to easily derive judgment aggregation results in a general logic $(\mathbf{L}, \vDash)$. Many past results essentially use these tools, and so they continue to hold in general logics.

To give the reader a feeling, suppose we want to generalise the introductory example where propositionwise majority voting generates the inconsistent judgment set $Y=\{a, a \rightarrow b, \neg b\}$. Note that $Y$ is minimal inconsistent: its proper subsets are consistent. Let us construct a similar inconsistency for any agenda $X \subseteq \mathbf{L}$ in a logic $(\mathbf{L}, \models)$ satisfying L1-L3 where $X$ has again a minimal inconsistent subset $Y$ with $|Y| \geq 3$. Note that $Y$ becomes consistent whenever one member is removed. So we can let each of the three equally-sized camps accept all but one proposition in $Y$, where the non-accepted proposition is a different one for each camp. By L3 (more precisely by Proposition 2(e)), we can extend a camp's accepted subset $B \subseteq Y$ to a complete and consistent judgment set $C \subseteq X$ of that camp. The resulting profile $\left(A_{1}, \ldots, A_{n}\right)$ contains fully rational judgment sets, and majority voting generates a judgment set $A \supseteq Y$. As $Y$ is inconsistent, so is $A$ by L2 (or Proposition 2(b)).

In Section 4 1, I give tools valid under L1-L3, and I argue that L1-L3 is an appropriate framework if the only rationality conditions of interest are consistency and completeness. In Section 4.2, I give additional tools under L1-L4, and I argue that L1-L4 may be appropriate to analyse the rationality condition of deductive closure.

\subsection{Judgment aggregation in L1-L3: rationality as completeness and consistency}

Suppose that the only rationality criteria of interest are completeness and consistency (and weak consistency), whose conjunction defines full rationality. That is, suppose we only ask whether or not the collective judgment set is consistent and whether or not it is complete; we do not ask whether it is deductive closed. (Note that if it is both consistent and complete, i.e. fully rational, then it is automatically deductively closed; see Proposition 1.) Under this premise, the results that one may want to derive involve no rationality condition except from consistency and/or completeness; examples are results on the (im)possibility of aggregation with fully rational outcomes, 
and characterisations of aggregation rules with consistent outcomes. Such results can usually be based on a general logic of type L1-L3 (plus perhaps L5); L4 plays no role and need not be assumed.

Let me justify this claim. Such results do not appeal directly to the entailment relation $\vDash$, but only to the consistency notion induced by $\vDash$ : they depend only on properties of the system $\mathcal{I}(\subseteq \mathcal{P}(\mathbf{L}))$ of inconsistent sets, regardless of which entailment relation $\vDash$ has induced the system $\mathcal{I}$. But what sort of properties of inconsistency (i.e. of $\mathcal{I}$ ) do such results depend on? The properties listed in Proposition 2 turn out to be all that is needed to prove many such results, including all results of Section 5 , Nehring and Puppe's (2004) characterisation of consistent voting by committees, Dietrich and List's (2004b) liberal paradox for judgment aggregation, and Dietrich and List's (2005) characterisation of consistent quota rules. So, as Proposition 2 requires only L1-L3 (plus perhaps L5), all of these results are valid in general logics of type L1-L3 (plus perhaps L5 if the agenda $X$ is infinite). Condition L4 would not have added anything. ${ }^{22}$ L1-L3 are also the conditions under which inconsistencies can be represented using a Venn diagram. ${ }^{23}$ Note that if entailment is of no independent interest then, although less natural in logic, one could reformulate the general logics model such that inconsistency is a primitive notion. A logic would then be defined not as a pair $(\mathbf{L}, \vDash)$ but as a pair $(\mathbf{L}, \mathcal{I})$, thereby loosing the notion of entailment and hence of deductive closure. ${ }^{24}$ Formally this approach is closely related to Wilson's (1975) aggregation model.

Proposition 2 (properties of inconsistency) Assume L1-L3. For any $A \subseteq \mathbf{L}$ and $p \in \mathbf{L}$,

(a) if $p, \neg p \in A$ then $A$ is inconsistent;

(b) if $A$ is inconsistent then so is any superset of $A$;

(c) if $A$ is consistent then so is any subset of $A$;

(d) if $A$ is consistent then $A \cup\{p\}$ or $A \cup\{\neg p\}$ is consistent;

(e) for every consistent judgment set $B \subseteq X$, there is a fully rational judgment set $C \subseteq X$ with $B \subseteq C$;

(f) $A$ is consistent if and only if $A^{*}$ is consistent, for any set $A^{*}$ arising from $A$ by replacing elements $q \in A$ by one or more $q$-variants $;{ }^{25}$

\footnotetext{
${ }^{22}$ L1-L4 imply no properties of the system of inconsistent sets $\mathcal{I}$ other than those already implied by L1-L3. That is, the set of conditions L1-L4 on $\vDash$ and the set of conditions L1-L3 on $\vDash$ imply exactly the same properties of $\mathcal{I}$ : they each imply that $\mathcal{I}$ satisfies the conditions I1-I3 defined in footnote 24 .

${ }^{23}$ Identify the worlds (points) of the Venn diagram with the consistent sets $A \subseteq \mathbf{L}$ containing a member of each pair $p, \neg p \in \mathbf{L}$, and identify each $p \in \mathbf{L}$ with the set of worlds containing $p$. Then, as desired for a Venn diagram, a set $A \subseteq \mathbf{L}$ is consistent if and only if the intersection of the sets of worlds corresponding to the propositions in $A$ is non-empty. In general, a Venn diagram can fully represent the logic's inconsistencies (i.e. $\mathcal{I}$ ) but not its entailments (i.e. $\vDash$ ).

${ }^{24}$ One then needs to impose the following conditions on $(\mathbf{L}, \mathcal{I})$ : (I1) for each $p \in \mathbf{L}$, we have $\{p, \neg p\} \in \mathcal{I}$; (I2) for all $A, B \subseteq \mathbf{L}$, if $A \in \mathcal{I}$ and $A \subseteq B$ then $B \in \mathcal{I}$; (I3) $\emptyset \notin \mathcal{I}$, and every $A \subseteq \mathbf{L}$ with $A \notin \mathcal{I}$ has a superset $B \subseteq \mathbf{L}$ with $B \notin \mathcal{I}$ containing a member of each pair $p, \neg p \in \mathbf{L}$. The conditions I1-I3 on $(\mathbf{L}, \mathcal{I})$ are equivalent to the conditions L1-L3 on $(\mathbf{L}, \models)$ : for any language $\mathbf{L}$, a system $\mathcal{I}$ $(\subseteq \mathcal{P}(\mathbf{L}))$ satisfies I1-I3 if and only if it is the set of inconsistent sets generated by some entailment relation $\vDash$ satisfying L1-L3 (if we even require L1-L4, $\vDash$ is unique, and given by $[A \vDash p$ if and only if $A \cup\{\neg p\} \in \mathcal{I}]$ ). While inconsistency can be defined in terms of entailment, the converse is impossible (unless one assumes L4): starting from $(\mathbf{L}, \mathcal{I})$ satisfying I1-I3, one cannot define $\vDash$ (without assuming L4), and hence deductive closure is undefinable.

${ }^{25} q^{*}$ is a $q$-variant (and $q$ a $q^{*}$-variant) if one of $q$ and $q^{*}$ is a $k$-fold negation of the other for some
} 
(g) every finite inconsistent set has a minimal inconsistent subset;

(h) given L5, every inconsistent set has a finite minimal inconsistent subset.

Note that by part (f) any double-negated proposition $\neg \neg p$ is essentially identical to $p$ with respect to inconsistencies: both stand in exactly the same inconsistency relations with other propositions.

Proof. (a) If $p, \neg p \in A$ then by L1-L2 $A \vDash p$ and $A \vDash \neg p$; so $A$ is inconsistent.

(b), (c): These claims follow from L2.

(d) If $A$ is consistent, then by L3 $A$ has a consistent superset $C \subseteq \mathbf{L}$ containing a member of each pair $p, \neg p \in \mathbf{L}$. As $C$ is a superset of either $A \cup\{p\}$ or $A \cup\{\neg p\}$, either of the latter sets is consistent by (c).

(e) Let $B \subseteq X$ be a consistent judgment set. By L3 $B$ has a consistent superset $D \subseteq \mathbf{L}$ containing a member of each pair $p, \neg p \in \mathbf{L}$. So the judgment set $C:=D \cap X$ is complete and by (c) consistent, and it satisfies $B \subseteq C$.

(f) Let $A^{*}$ be as specified. I show that any set $S \subseteq \mathbf{L}$ is consistent if and only if the set $\widetilde{S}:=\{q \in \mathbf{L}: q$ is a variant of some $r \in S\}$ is consistent. Using this, the claim is equivalent to the claim that $\widetilde{A}$ is consistent if and only if $\widetilde{A^{*}}$ is, which holds by $\widetilde{A}=\widetilde{A^{*}}$.

Consider any $S \subseteq \mathbf{L}$. If $\widetilde{S}$ is consistent, then so is $S$ by $S \subseteq \widetilde{S}$ and (c). Now let $S$ be consistent. Then, by L3, $S$ has a consistent superset $T \subseteq \mathbf{L}$ containing a member of each pair $q, \neg q \in \mathbf{L}$. I show that $\widetilde{S} \subseteq T$, which by (c) implies that $\widetilde{S}$ is consistent, as desired. To show $\widetilde{S} \subseteq T$, I have to prove that, for any $q \in S, T$ contains all $q$-variants, i.e. all $q^{*} \in \mathbf{L}$ such that for some even $k \in\{0,2,4, \ldots\}$ (i) $q^{*}$ is the $k$-fold negation of $q$ or $q$ is the $k$-fold negation of $q^{*}$. I only show case (i), as case (ii) can be shown analogously. Of course $q \in B$. By $q \in B$, we have $\neg q \notin B$ by (a), and hence $\neg \neg q \in B$ since $B$ contains one of $\neg q, \neg \neg q$. Repeating this argument, one finds $\neg \neg \neg \neg q \in B$, then $\neg \neg \neg \neg \neg \neg q \in B$, etc., as claimed.

(g) Let $B \subseteq \mathbf{L}$ be any finite inconsistent set. Among all inconsistent subsets of $B$, choose a minimal one (with respect to inclusion); there exists one since $B$ is finite. This set is minimal inconsistent.

(h) Any inconsistent set has by L5 a finite inconsistent subset, and hence by (g) a finite minimal inconsistent subset.

\subsection{Judgment aggregation in L1-L4: analysing deductive closure}

In addition to completeness and consistency, it may be interesting to analyse the rationality condition of deductive closure; this analysis may require the additional assumption of L4 (non-paraconsistency). One motivation for studying deductive closure can arise from the various impossibility theorems. Let me explain how. These theorems tell us that fully rational aggregation is often unrealistic. But if collective judgments cannot be fully rational, what weaker form of rationality should we aim at? There are (at least) two approaches:

- Relaxing completeness, but keeping consistency and deductive closure. Here, the collective abstains from a decision on certain pairs $p, \neg p$, but otherwise forms judgments that are not only consistent, but also deductively closed, i.e. whenever

even number $k \in\{0,2,4, \ldots\}$. For instance, $q$ and $\neg \neg q$ are $q$-variants. 
a proposition $p \in X$ follows from the collectively accepted propositions, $p$ is also accepted. It is then interesting to analyse which aggregation rules generate consistent and deductively closed judgment sets. As seen in Proposition 3 below, some tools to analyse deductive closure hold for all logics of type L1-L3, but many others require a logic of type L1-L4. Dietrich and List's (2005) characterisation of consistent and deductively closed quota rules holds in logics of type L1-L4 (plus L5 if the agenda $X$ is infinite).

- Relaxing consistency to weak consistency, but keeping completeness and deductive closure. Here, the collective accepts exactly one member of each pair $p, \neg p$, in a deductively closed but perhaps not consistent way. This form of restricted rationality exists only in logics violating L4, because, under L1-L4, by keeping completeness, weak consistency and deductive closure one actually keeps consistency (see Proposition 1). But the escape route may be an interesting option in logics violating L4, i.e. in paraconsistent logics with various degrees of consistency. In some real situations, relaxing consistency may even be the only feasible collective rationality relaxation, because completeness cannot be given up when a decision on each pair $p, \neg p \in X$ is strictly needed. As L4 should not be imposed, the tools (d)-(f) in Proposition 3 are unavailable.

Proposition 3 (properties of entailment) Assume L1-L3. For any $A \subseteq \mathbf{L}$ and $p \in \mathbf{L}$,

(a) if $p \in A$ then $A \vDash p$;

(b) if $A \vDash p$ then $A \cup\{\neg p\}$ is inconsistent; in particular, $\emptyset$ entails only tautologies;

(c) if $A \vDash p$ and $A$ is consistent then $A \cup\{p\}$ is consistent;

(d) given L4, each inconsistent set entails every proposition;

(e) given $L 4, A \vDash p$ if and only if $A \cup\{\neg p\}$ is inconsistent.

(f) given $L 4, A \vDash p$ if and only if $A^{*} \vDash p^{*}$, for any $p$-variant $p^{*}$ and any set $A^{*}$ arising from $A$ by replacing elements $q \in A$ by one or more $q$-variants. ${ }^{26}$

Under L1-L4 the entailment relation $\vDash$ can be retrieved from the inconsistency notion using (e); see also footnote 24. By part (f), under L1-L4 double-negations

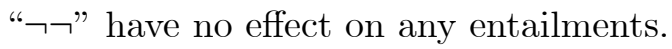

Proof. (a) follows from L1 and L2.

(b) If $A \vDash p$, then $A \cup\{\neg p\}$ is inconsistent since $A \cup\{\neg p\} \vDash p$ by L2 and $A \cup\{\neg p\} \vDash$ $\neg p$ by (a).

(c) Assume $A \vDash p$ where $A$ is consistent. By L3, $A \cup\{p\}$ or $A \cup\{\neg p\}$ is consistent. As $A \cup\{\neg p\}$ is inconsistent by (b), $A \cup\{p\}$ is consistent.

(d) For any inconsistent set $A \subseteq \mathbf{L}$ and any $p \in \mathbf{L}$, by L2 $A \cup\{\neg p\}$ is inconsistent, and hence by L $4 A \vDash p$.

(e) One direction follows from (b), the other one from L4.

(f) Let $A^{*}$ and $p^{*}$ be as specified. By (e) and L4, $A \vDash p$ if and only if $A \cup\{\neg p\}$ is inconsistent, and $A^{*} \vDash p^{*}$ if and only if $A^{*} \cup\left\{\neg p^{*}\right\}$ is inconsistent. So the claim follows from Proposition 2(f).

With Propositions 2 and 3 in place, I can now prove Proposition 1 on the relations between the various rationality conditions on judgment sets.

${ }^{26}$ see footnote 25 
Proof of Proposition 1. Assume L1-L3 and let $A \subseteq X$ be any judgment set.

(a) If $A$ is consistent then it is weakly consistent by Proposition 2(c). Now assume $A$ is deductively closed. Of course, consistency still implies weak consistency. Conversely, suppose $A$ is not consistent. So $A$ entails each $p \in X$ by Proposition 3(d). Hence, by deductive closure, $A=X$. So $A$ is not weakly consistent.

(b) First, let $A \subseteq X$ be fully rational. Then $A$ is complete, and Proposition 2(c) weakly consistent. To prove deductive closure, consider any $p \in X$ such that $A \vDash p$. By $A \vDash p$, the set $A \cup\{\neg p\}$ is inconsistent by Proposition 3(b). First assume $p$ is not a negated proposition; then $p, \neg p \in X$ by the definition of agendas. Since $A$ is consistent, $A \neq A \cup\{\neg p\}$, and hence $\neg p \notin A$, which implies $p \in A$ by $A$ 's completeness. Now assume $p$ is a negated proposition, say $p=\neg q$; then $q, \neg q \in X$ by the definition of agendas. Since $A \cup\{\neg p\}=A \cup\{\neg \neg q\}$ is inconsistent, so is $A \cup\{q\}$ by Proposition 2(f). So, as $A$ is consistent, $A \neq A \cup\{q\}$, and hence $q \notin A$, which implies $\neg q=p \in A$ by $A$ 's completeness.

Now assume also L4 and let $A$ be complete, weakly consistent and deductively closed. Assume for contradiction that $A$ is not fully rational. Then, as $A$ is complete, it is not consistent. So, by (a) and $A$ 's deductive closure, $A$ is not weakly consistent, contradicting the assumption.

\section{An impossibility result in general logics}

Let $(\mathbf{L}, \vDash)$ be any logic of type L1-L3. Using the tools of Proposition 2, I now present an impossibility result that generalises the binary case of Pauly and van Hees' (2004) impossibility theorem on systematicity, which in turn strengthens List and Pettit's (2002) original theorem. Unlike these theorems, the present result captures the standard judgment aggregation examples under an adequate representation of conditional statements. The result also relates to a theorem in the property space framework by Nehring and Puppe (2002), as explained below.

While in preference aggregation Arrow's independence of irrelevant alternatives leads (together with mild other conditions) into dictatorship, the earlier-defined independence condition, introduced in judgment aggregation by Pauly and van Hees (2004), does so only for quite special agendas. This is demonstrated, for instance, by theorems by Pauly and van Hees (2004) and Dietrich (2004). But impossibilities for more general agendas have been obtained by using a more demanding condition:

Systematicity. For any propositions $p, p^{*} \in X$ and profiles $\left(A_{1}, \ldots, A_{n}\right),\left(A_{1}^{*}, \ldots\right.$, $\left.A_{n}^{*}\right) \in \operatorname{Domain}(F)$, if [for all individuals $i, p \in A_{i}$ if and only if $p^{*} \in A_{i}^{*}$ ] then $\left[p \in F\left(A_{1}, \ldots, A_{n}\right)\right.$ if and only if $\left.p^{*} \in F\left(A_{1}^{*}, \ldots, A_{n}^{*}\right)\right]$.

Taking $p=p^{*}$ yields exactly the independence condition. Systematicity is equivalent to the existence of a function $M:\{0,1\}^{n} \rightarrow\{0,1\}$ (the "universal decision method") such that, for every proposition $p \in X$ and profile $\left(A_{1}, \ldots, A_{n}\right) \in$ $\operatorname{Domain}(F), F\left(A_{1}, \ldots, A_{n}\right)(p)=M\left(A_{1}(p), \ldots, A_{n}(p)\right)$. Here, for any $A \subseteq X, A(p)$ is defined as 1 if $p \in A$ and as 0 if $p \notin A$. Systematic rules include majority rule $\left(M\left(t_{1}, \ldots, t_{n}\right)=1\right.$ if and only if $\left.t_{1}+\ldots+t_{n}>n / 2\right)$, unanimity rule $\left(M\left(t_{1}, \ldots, t_{n}\right)=1\right.$ if and only if $\left.t_{1}=\ldots=t_{n}=1\right)$, dictatorial rules $\left(M\left(t_{1}, \ldots, t_{n}\right)=t_{j}\right.$, where $j$ is the dictator $)$, inverse dictatorial rules $\left(M\left(t_{1}, \ldots, t_{n}\right)=1-t_{j}\right.$, where $j$ is the inverse dictator $)$, 
oligarchic rules, inverse oligarchic rules, etc. Systematicity differs from independence in that the decision method $M$ may not depend on $p$.

To obtain a result in general logics, the agenda $X$ should not be required to contain particular propositions (formable only in certain logics), but rather to display certain relations between its propositions. The following agenda conditions turn out to be appropriate. Let us call a set $Y \subseteq \mathbf{L}$ minimal inconsistent if it is inconsistent and every proper subset of $Y$ is consistent; and let us write $Y\urcorner:=\{\neg p: p \in Y\}$.

Definition 2 The agenda $X$ is minimally connected if

(i) $X$ has a minimal inconsistent subset $Y$ such that $|Y| \geq 3$; and

(ii) $X$ has a minimal inconsistent subset $Y$ such that $(Y \backslash Z) \cup Z\urcorner$ is consistent for some set $Z \subseteq Y$ of even size.

$X$ is asymmetric if $X$ has a subset $S$ such that one but not the other one of the sets $S, S\urcorner$ is consistent.

I will show that these two agenda conditions are together sufficient and essentially necessary for a general impossibility result to hold. First, however, let me discuss these conditions and assess their strength. Agendas (decision problems) without any logical interconnections (except those between propositions and their negations) are of course neither minimally connected nor asymmetric. Examples are agendas consisting only of atomic propositions and their negations (say, in classical propositional logic), and the agendas $X=\{a, \neg a, S(a), \neg S(a)\}$ and $X=\{a, \neg a, S(a \stackrel{\text { mat }}{\rightarrow} b), \neg S(a \stackrel{\text { mat }}{\rightarrow} b), b, \neg b\}$ where $S$ means "it is desirable that" an $a$ and $b$ are atomic propositions, say in the modal logic $K_{\Delta}$ with $\Delta$ containing only the extensibility condition (see Section 3.3).

However, once the agenda displays logical interconnections (beyond the trivial ones within pairs $p, \neg p \in X$ ), the agenda will typically be both minimally connected and asymmetric. In particular, this is so for all standard examples of judgment aggregation, whether conditional statements are modelled with material conditionals (problematic) or with strict or subjunctive conditionals (adequate). For instance, an agenda $X$ containing distinct atomic propositions $a, b$ and their conjunction $a \wedge b$ (as in List and Pettit's 2002 theorem) is minimally connected (take $Y=\{a, b, \neg(a \wedge b)\}$ in (i) and (ii), and $Z=\{a, b\}$ in (ii)) and asymmetric (take $S=\{a, \neg(a \wedge b)\}$ ). Also an agenda $X$ containing distinct atomic propositions $a, b$ and a (material or non-material) implication $a \rightarrow b$ is minimally connected (take $Y=\{a, a \rightarrow b, \neg b\}$ in (i) and (ii), and $Z=\{a, \neg b\}$ in (ii)) and asymmetric (take $S=\{a, a \rightarrow b\}$ if $\rightarrow$ is a material conditional and $S=\{a, a \rightarrow b, \neg b\}$ otherwise). Moreover, the agenda $X_{C}$ for representing a weak preference aggregation problem with set of alternatives $C$ (see Section 3.4) is also minimally connected and asymmetric, as shown below.

Condition (i) can be regarded as a lower bound on the complexity of (the logical interconnections in) the decision problem. Denote the size of the largest minimal inconsistent set $Z \subseteq X$ by $z$, interpreted as a measure of the complexity of the decision problem $X$. Condition (i) requires that $z \geq 3$. This excludes the case $z=1$, which arises when $X$ contains only tautologies and contradictions, and the case $z=2$, which arises for still quite simple agendas like those consisting of atomic propositions and their negations (the only minimal inconsistent sets being then pairs $\{p, \neg p\} \subseteq X$ ).

Condition (ii) is harder to interpret, though I will later point out an algebraic approach. Nevertheless, examples such as the ones above illustrate that (ii) holds for most agendas with (non-trivial) logical interconnections. In (ii), it is essential that $Z$ 
has even size: allowing $|Z|=1$ renders the condition always true (because every minimal inconsistent set $Y$ becomes consistent by negating exactly one of its members), and demanding only that $|Z| \geq 2$ is not sufficient to generate the impossibility. ${ }^{27}$

Finally, asymmetry is also a mild requirement because the opposite - the symmetry condition that any $S \subseteq X$ is consistent if and only $S\urcorner$ is consistent - is obviously a special case.

Let us call an aggregation rule $F$ regular if it satisfies universal domain and collective rationality; these conditions are, like dictatorship, defined in Section 2.3.

Theorem 1 If the agenda $X$ is minimally connected and asymmetric, every regular and systematic aggregation rule is dictatorial.

This result, proven further below, obviously generalises List and Pettit's (2002) theorem: dictatorship replaces non-anonymity, and on the agenda side we use weaker interconnections and not necessarily classical propositional logic. Pauly and van Hees' (2004) Theorem 4 is a different strengthening of List and Pettit's theorem: it allows more than two degrees of acceptance (in addition to using dictatorship like I do in Theorem 1). In the binary case of exactly two degrees of acceptance (as in the present paper), their Theorem 4 follows from Theorem 1 because their agenda is minimally connected and asymmetric. ${ }^{28}$ Nehring and Puppe (2002) prove a theorem in the property space model related to Theorem 1, which implies the following result on logics of type L1-L3: under an even weaker agenda assumption (only part (i) of minimal connectedness), each regular, systematic and monotone aggregation rule is dictatorial (in fact, they use independence and neutrality instead of systematicity). ${ }^{29}$

As an application, consider the agendas introduced in Section 3.4 to represent a preference aggregation problem with set of options $C=\left\{c_{1}, \ldots, c_{k}\right\}$ and set of desirable rationality conditions $\mathcal{R}$. I have distinguished between the agenda $X_{C}:=$ $\left\{c R c^{\prime}, \neg c R c^{\prime}: c, c^{\prime} \in C\right\}$, where the rationality conditions are exogenously given (as in standard preference aggregation), and the agenda $X_{C, \mathcal{R}}:=\left\{c R c^{\prime}, \neg c R c^{\prime}: c, c^{\prime} \in\right.$

\footnotetext{
${ }^{27}$ The agenda $X=\{a, \neg a, b, \neg b, b \stackrel{\text { mat }}{\leftrightarrow} a, \neg(b \stackrel{\text { mat }}{\leftrightarrow} a)\}$ in classical propositional logic violates condition (ii): check that, whenever we consider a minimal inconsistent set $Y \subseteq X$ (e.g. $Y=\{a, b, \neg(a \stackrel{\text { mat }}{\leftrightarrow} b)\})$, $Y$ stays inconsistent if we negate exactly two of its members. However, $X$ would satisfy (ii) if (ii) allowed that $|Z|=3: \quad Y=\{a, b, \neg(a \stackrel{\text { mat }}{\leftrightarrow} b)\}$ is minimal inconsistent, but if we negate all three propositions we obtain the consistent set $\{\neg a, \neg b, a \stackrel{\text { mat }}{\leftrightarrow} b\}$. In Theorem 1 we cannot weaken (ii) by allowing $|Z|=3$ : if $n=3$ and $X$ is the mentioned agenda, the systematic rule with universal domain that accepts those propositions accepted by an odd number of individuals generates consistent judgment sets. The reader might check this by hand, or inspect case 3 of the proof of Proposition $2^{*}$ in conjunction with Remark 1.

${ }^{28}$ Consider the binary case of Pauly and van Hees' Theorem 4. This theorem then assumes (just like List and Pettit's theorem) that $X$ contains distinct atomic propositions $a, b$ and $a \wedge b, \neg(a \wedge b)$. A subtlety is that Pauly and van Hees do not like me build into the definition of an agenda to be a union of pairs $\{p, \neg p\}$ with $p$ not a negated proposition. So in their Theorem $4 X$ need not contain $\neg a$ and $\neg b$. However, to any agenda $X$ in their sense corresponds one in my sense, obtained by adding to $X \neg p$ for each $p$ already in $X$, and then cancelling out double-negations $\neg \neg$. The regular systematic aggregation rule assumed in their Theorem 4 induces a unique regular systematic aggregation rule for the corresponding agenda in my sense, and so we can apply Theorem 1 to deduce dictatorship.

${ }^{29}$ That is, Theorem 1 stays true if we add monotonicity but use (i) as the only agenda condition. For this modified Theorem 1, Theorem 2 still holds. Analogously, Theorem $1^{*}$ stays true if we add monotonicity but use $\left(i^{*}\right)$ as the only agenda condition; and, for this modified Theorem $1^{*}$, Theorem $2^{*}$ still holds. All this is clear from the proofs.
} 
$C\} \cup\{r, \neg r: r \in \mathcal{R}\}$, where the rationality conditions are endogenous, i.e. also under decision (the logic $(\mathbf{L}, \vDash)$ of each agenda is also defined in Section 3.4). For instance, suppose that $\mathcal{R}$ is the set of conditions defining a weak order or a linear order:

$$
\begin{aligned}
& \mathcal{R}=\left\{r_{1}, r_{2}\right\} \text { (weak order) or } \mathcal{R}=\left\{r_{1}, r_{2}, r_{3}\right\} \text { (linear order), where } \\
& r_{1} \text { is } \forall v_{1} \forall v_{2}\left(v_{1} R v_{2} \vee v_{2} R v_{1}\right)(\text { completeness), } \\
& r_{2} \text { is } \forall v_{1} \forall v_{2} \forall v_{3}\left(\left(v_{1} R v_{2} \wedge v_{2} R v_{3}\right) \rightarrow v_{1} R v_{3}\right) \text { (transitivity), } \\
& r_{3} \text { is } \forall v_{1} \forall v_{2}\left(\left(v_{1} R v_{2} \wedge v_{2} R v_{1}\right) \rightarrow v_{1}=v_{2}\right) \text { (antisymmetry). }
\end{aligned}
$$

Then, if $C$ contains at least three options, the agenda $X_{C}$ is minimally connected (take $Y=\left\{c_{1} R c_{2}, c_{2} R c_{3}, \neg c_{1} R c_{3}\right\}$ in (i) and (ii)) and asymmetric (take $S=\left\{c_{1} R c_{1}\right\}$ ). Interestingly, the agenda $X_{C, \mathcal{R}}$ is minimally connected and asymmetric even for two options (take $Y=\left\{\neg c_{1} R c_{2}, \neg c_{2} R c_{1}, \forall v_{1} \forall v_{2}\left(v_{1} R v_{2} \vee v_{2} R v_{1}\right)\right\}$ in (i) and(ii), and $S=$ $Y)$. So Theorem 1 has the following implication.

Corollary 1 Let the set of rationality conditions $\mathcal{R}$ satisfy (2).

(a) (exogenous rationality) If there are $|C| \geq 3$ options, any regular and systematic aggregation rule for the agenda $X_{C}$ is dictatorial.

(b) (endogenous rationality) If there are $|C| \geq 2$ options, any regular and systematic aggregation rule for the agenda $X_{C, \mathcal{R}}$ is dictatorial.

Part (a) is not much surprise in the light of Arrow's theorem (see also results by List and Pettit 2004 and Nehring 2003). ${ }^{30}$ Part (b) shows that endogenising the rationality conditions does not help overcoming the impossibility: worse, it extends the impossibility to the case of only two options.

Before proving Theorem 1, let us ask whether our agenda conditions - asymmetry and minimal connectedness - are essential for the impossibility. Asymmetry is indispensable, as otherwise inverse dictatorships (defined on the universal domain by $F\left(A_{1}, \ldots, A_{n}\right)=\left\{\neg p: p \in A_{j}\right\}$ for some inverse dictator $j$ ) generate consistent judgment sets. Condition (i) is also indispensable if $n \geq 3$ and

$\left(^{*}\right)$ the agenda $X$ is finite or the logic satisfies L5 (compactness),

since without (i) majority voting generates consistent judgment sets. As Ron Holzman indicated to me, also condition (ii) is indispensable, still assuming $n \geq 3$ and (*). Dokow and Holzman (2005) independently introduced an algebraic condition, which turned out to be equivalent to condition (ii) (if $X$ is finite). Dokow and Holzman consider a finite agenda $X=\left\{p_{1}, \neg p_{1}, \ldots, p_{k}, \neg p_{k}\right\}$ and call a a vector of truth values $\left(t_{1}, \ldots, t_{k}\right) \in\{0,1\}^{k}$ a feasible evaluation in case the corresponding judgment set (consisting of all $p_{j} \in X$ with $t_{j}=1$ and all $\neg p_{j} \in X$ with $t_{j}=0$ ) is consistent. Ron Holzman pointed out to me that the agenda condition (ii) holds if and only if the set $B \subseteq\{0,1\}^{k}$ of feasible evaluations is not an affine subspace of $\{0,1\}^{k}$, which is the central algebraic condition analysed by Dokow and Holzman. ${ }^{31}$

In summary, we have the following result, whose formal proof is given later.

\footnotetext{
${ }^{30}$ While Arrow's independence of irrelevant alternatives is much weaker than our systematicity, note that his impossibility theorem also requires a Pareto condition.

${ }^{31} \mathrm{~A}$ set $B \subseteq\{0,1\}^{k}$ is a linear subspace if it is closed under addition in the vector space $\{0,1\}^{k}$ over the binary field $\{0,1\}$ (with addition defined modulo 2 , e.g. $(1,1, \ldots, 1)+(1,0, \ldots, 0)=(0,1, \ldots, 1)$ ). $B \subseteq\{0,1\}^{k}$ is an affine subspace if $B=\left\{b^{*}+c: b^{*} \in B^{*}\right\}$ for some linear subspace $B^{*} \subseteq\{0,1\}^{k}$ and some fixed vector $c \in\{0,1\}^{k}$. Based on their algebraic condition, Dokow and Holzman derive an impossibility theorem that builds on an impossibility theorem by Nehring and Puppe (2002).
} 
Theorem 2 The converse of Theorem 1 holds whenever (*) holds and $n \geq 3$ (and $X$ contains a contingent proposition).

So, in important cases, the agenda conditions of Theorem 1 are indeed minimal. Instead of proving Theorems 1 and 2, I will prove slightly stronger results. More precisely, I will present a generalised definition of "minimally connected", in which I weaken the conditions (i) and (ii) such that Theorems 1 and 2 still hold and in Theorem 2 we can now drop the restriction $(*)$.

Definition 3 The agenda $X$ is minimally connected (second sense) if

$\left(i^{*}\right) X$ has an inconsistent subset $Y$ with pairwise disjoint subsets $Z_{1}, Z_{2}, Z_{3}$ such that $\left(Y \backslash Z_{j}\right) \cup Z_{j}$ is consistent for any $j \in\{1,2,3\}$; and

$\left(i i^{*}\right) X$ has an inconsistent subset $Y$ with pairwise disjoint subsets $Z_{1}, Z_{2}, Z_{3}$ such that $\left.\left(Y \backslash\left(Z_{j} \cup Z_{k}\right)\right) \cup\left(Z_{j} \cup Z_{k}\right)\right\urcorner$ is consistent for any distinct $j, k \in\{1,2,3\}$.

Remark 1 Minimal connectedness in the first sense implies minimal connectedness in the second sense, and the two are equivalent under $\left({ }^{*}\right)$.

Proof. For any negated proposition $q=\neg p \in X$, let $\neg q$ stand for $p$ rather than for $\neg \neg p .^{32}$

1. I first show that (i) implies $\left(i^{*}\right)$, and (ii) implies (ii*). If (i) holds, then $\left(i^{*}\right)$ follows by taking $Y$ to be as in (i) and letting $Z_{1}, Z_{2}, Z_{3} \subseteq Y$ be disjoint singletons. Now assume (ii) holds. Let $Y$ be as in (ii), and let $Z$ be a smallest even-sized subset of $Y$ such that $(Y \backslash Z) \cup Z\urcorner$ is consistent. Consider any partition of $Z$ into sets $Z_{1}, Z_{2}, W \subseteq Z$, where $Z_{1}$ and $Z_{2}$ are singletons. Then (ii*) can be shown to hold by considering the set $\left.Y^{*}:=(Y \backslash W) \cup W\right\urcorner$ with pairwise disjoint subsets $Z_{1}, Z_{2}$, and $Z_{3}:=W^{\urcorner}$.

2. Now assume $(*)$. I show that $\left(\mathrm{i}^{*}\right)$ implies (i), and (ii*) implies (ii). If (i*) holds, there is an inconsistent set $Y \subseteq X$ with pairwise disjoint subsets $Z_{1}, Z_{2}, Z_{3}$ such that each $\left(Y \backslash Z_{j}\right) \cup Z_{j}, j=1,2,3$, is consistent. By $\left(^{*}\right) Y$ has a finite minimal inconsistent subset $Y^{*}$. Each $Z_{i}, i=1,2,3$, must have a non-empty intersection with $Y^{*}$, as otherwise $\left(Y \backslash Z_{j}\right) \cup Z_{j}^{\urcorner}$would contain the (inconsistent) set $Y^{*}$. So $\left|Y^{*}\right| \geq 3$, proving (i). Now assume (ii*). Then there are an inconsistent set $Y \subseteq X$ with pairwise disjoint subsets $Z_{1}, Z_{2}, Z_{3}$ such that $\left.\left(Y \backslash\left(Z_{j} \cup Z_{k}\right)\right) \cup\left(Z_{j} \cup Z_{k}\right)\right\urcorner$ is consistent for any distinct $j, k \in\{1,2,3\}$. By $\left(^{*}\right) Y$ has a finite minimal inconsistent subset $Y^{*}$. Put $Z_{j}^{*}:=Z_{j} \cap Y^{*}$ for each $j=1,2,3$. Note that, regardless of how many of the sets $Z_{1}^{*}, Z_{2}^{*}, Z_{3}^{*}$ have even size, there must exist distinct indices $j, k \in\{1,2,3\}$ such that the union $Z:=Z_{j}^{*} \cup Z_{k}^{*}$ has even size. The set $\left(Y^{*} \backslash Z\right) \cup Z^{\urcorner}$is consistent, as it is a subset of $\left.\left(Y \backslash\left(Z_{j} \cup Z_{k}\right)\right) \cup\left(Z_{j} \cup Z_{k}\right)\right\urcorner$. So (ii) holds.

As desired, this second sense of "minimally connected" yields the following stronger versions of Theorems 1 and 2 .

Theorem 1* If the agenda $X$ is minimally connected (in the second sense) and asymmetric, every regular and systematic aggregation rule is dictatorial.

\footnotetext{
${ }^{32}$ More precisely, when I use $\neg$ in front of some $p \in X$ I mean a modified negation symbol $\sim$, where $\sim p:=q$ if $p=\neg q$ for some $q$, and $\sim p:=\neg p$ otherwise. $p$ and $\neg \neg p$ are essentially identical in the sense of Proposition 2(f), but only $p$ is contained in $X$ as $X$ contains no double-negated propositions.
} 
Theorem $2^{*}$ The converse of Theorem $1^{*}$ holds whenever $n \geq 3$ (and $X$ contains a contingent proposition).

I now prove these theorems, which imply Theorems 1 and 2 by Remark 1 . As in the proof of Remark 1, I will make extensive (implicit) use of L1-L3 and Proposition 2 ; and I will again use the mentioned convention on double-negations.

Proof of Theorem $1^{*}$. Put $N:=\{1, \ldots, n\}$. Assume $X$ is minimally connected (second sense) and asymmetric, and $F$ is regular and systematic. Let $\mathcal{W}$ be the set of all coalitions $C \subseteq N$ for which there exists $p \in X$ and $\left(A_{1}, \ldots, A_{n}\right) \in \operatorname{Domain}(F)$ such that $\left\{i: p \in A_{i}\right\}=C$ and $p \in F\left(A_{1}, \ldots, A_{n}\right)$. Using systematicity, it follow that $F\left(A_{1}, \ldots, A_{n}\right)=\left\{p \in X:\left\{i: p \in A_{i}\right\} \in \mathcal{W}\right\}$. Call the members of $\mathcal{W}$ "winning coalitions". For any consistent set $Z \subseteq X$, let $A_{Z}$ be some consistent and complete judgment set such that $Z \subseteq A_{Z}$. (The existence of $A_{Z}$ is ensured by part (e) of Proposition 2.)

Claim 1. $N \in \mathcal{W}$, and, for every coalition $C \subseteq N, C \in \mathcal{W}$ if and only if $N \backslash C \notin \mathcal{W}$.

The second part of the claim follows from collective rationality together with universal domain. Now assume $N \notin \mathcal{W}$. By asymmetry, $X$ has a consistent subset $C$ such that $C\urcorner$ is inconsistent. Let $\left(A_{1}, \ldots, A_{n}\right)$ be the profile for which $A_{i}=A_{C}$ for all $i \in N$. As $N \notin \mathcal{W}, F\left(A_{1}, \ldots, A_{n}\right)$ contains no element of $C$. So $\left.C\right\urcorner \subseteq F\left(A_{1}, \ldots, A_{n}\right)$, violating the consistency of $F\left(A_{1}, \ldots, A_{n}\right)$.

Claim 2. There exists no partition of $N$ into three winning coalitions $C_{1}, C_{2}, C_{3}$.

Assume for contradiction that $C_{1}, C_{2}, C_{3}$ is a partition of $N$ with $C_{1}, C_{2}, C_{3} \in \mathcal{W}$. Let $Y, Z_{1}, Z_{2}, Z_{3}$ be as in (ii*). Consider the profile $\left(A_{1}, \ldots, A_{n}\right)$ defined by

$$
A_{i}:= \begin{cases}A_{\left.\left(Y \backslash\left(Z_{2} \cup Z_{3}\right)\right) \cup\left(Z_{2} \cup Z_{3}\right)\right\urcorner} & \text { if } i \in C_{1} \\ A_{\left(Y \backslash\left(Z_{1} \cup Z_{3}\right)\right) \cup\left(Z_{1} \cup Z_{3}\right)} & \text { if } i \in C_{2} \\ A_{\left(Y \backslash\left(Z_{1} \cup Z_{2}\right)\right) \cup\left(Z_{1} \cup Z_{2}\right)} & \text { if } i \in C_{3} .\end{cases}
$$

We have $Z_{1} \subseteq F\left(A_{1}, \ldots, A_{n}\right)$ as $C_{1} \in \mathcal{W}, Z_{2} \subseteq F\left(A_{1}, \ldots, A_{n}\right)$ as $C_{2} \in \mathcal{W}, Z_{3} \subseteq$ $F\left(A_{1}, \ldots, A_{n}\right)$ as $C_{3} \in \mathcal{W}$, and $Y \backslash\left(Z_{1} \cup Z_{2} \cup Z_{3}\right) \subseteq F\left(A_{1}, \ldots, A_{n}\right)$ as $N \in \mathcal{W}$ by claim 1. So $Y \subseteq F\left(A_{1}, \ldots, A_{n}\right)$, violating consistency.

Claim 3. For any coalitions $C, C^{*} \subseteq N$, if $C \in \mathcal{W}$ and $C \subseteq C^{*}$ then $C^{*} \in \mathcal{W}$.

Assume for contradiction that $C, C^{*} \subseteq N$ with $C \subseteq C^{*}, C \in \mathcal{W}$ and $C^{*} \notin \mathcal{W}$. Put $C_{1}:=C^{*} \backslash C$ and $C_{2}:=N \backslash C^{*}$. Then $C_{2} \in \mathcal{W}$ by claim 1. As $\left\{C, C_{1}, C_{2}\right\}$ is a partition of $N$, we have $C_{1} \notin \mathcal{W}$ by claim 2, and hence $N \backslash C_{1}=C \cup C_{2} \in \mathcal{W}$ by claim 1. Let $Y, Z_{1}, Z_{2}, Z_{3}$ be as in (i*). Consider first the profile $\left(A_{1}, \ldots, A_{n}\right)$ defined by

$$
A_{i}:= \begin{cases}A_{\left(Y \backslash Z_{1}\right) \cup Z_{1}} & \text { if } i \in C \\ A_{\left(Y \backslash Z_{2}\right) \cup Z_{2}^{\urcorner}} & \text {if } i \in C_{2} \\ A_{\left(Y \backslash Z_{3}\right) \cup Z_{3}^{\urcorner}} & \text {if } i \in C_{1} .\end{cases}
$$

We have $Z_{1} \subseteq F\left(A_{1}, \ldots, A_{n}\right)$ as $C \in \mathcal{W}, Z_{2} \subseteq F\left(A_{1}, \ldots, A_{n}\right)$ as $C_{2} \in \mathcal{W}, Z_{3} \subseteq$ $F\left(A_{1}, \ldots, A_{n}\right)$ as $C \cup C_{2} \in \mathcal{W}$, and $Y \backslash\left(Z_{1} \cup Z_{2} \cup Z_{3}\right) \subseteq F\left(A_{1}, \ldots, A_{n}\right)$ as $N \in \mathcal{W}$ by claim 1. In summary, we have $\left.\left(Y \backslash\left(Z_{1} \cup Z_{2}\right)\right) \cup\left(Z_{1} \cup Z_{2}\right)\right\urcorner \subseteq F\left(A_{1}, \ldots, A_{n}\right)$. So the set $\left.\left(Y \backslash\left(Z_{1} \cup Z_{2}\right)\right) \cup\left(Z_{1} \cup Z_{2}\right)\right\urcorner$ is consistent.

Using this consistency we now construct the following new profile $\left(A_{1}, \ldots, A_{n}\right)$ :

$$
A_{i}:= \begin{cases}A_{\left(Y \backslash Z_{1}\right) \cup Z_{1}} & \text { if } i \in C \\ A_{\left(Y \backslash Z_{2}\right) \cup Z_{2}} & \text { if } i \in C_{2} \\ A_{\left(Y \backslash\left(Z_{1} \cup Z_{2}\right)\right) \cup\left(Z_{1} \cup Z_{2}\right)} & \text { if } i \in C_{1} .\end{cases}
$$


We now have $Z_{2} \subseteq F\left(A_{1}, \ldots, A_{n}\right)$ as $C \in \mathcal{W}, Z_{1} \subseteq F\left(A_{1}, \ldots, A_{n}\right)$ as $C_{2} \in \mathcal{W}$, and $Y \backslash\left(Z_{1} \cup Z_{2}\right) \subseteq F\left(A_{1}, \ldots, A_{n}\right)$ as $N \in \mathcal{W}$ by claim 1 . So $Y \subseteq F\left(A_{1}, \ldots, A_{n}\right)$, violating consistency.

Claim 4. For any coalitions $C, C^{*} \subseteq N$, if $C, C^{*} \in \mathcal{W}$ then $C \cap C^{*} \in \mathcal{W}$.

Consider any $C, C^{*} \in \mathcal{W}$, and assume for contradiction that $C_{1}:=C \cap C^{*} \notin \mathcal{W}$. Put $C_{2}:=C^{*} \backslash C$ and $C_{3}:=N \backslash C^{*}$. Let $Y, Z_{1}, Z_{2}, Z_{3}$ be as in part (i*) of the agenda assumption. Noting that $C_{1}, C_{2}, C_{3}$ form a partition of $N$, we define the profile $\left(A_{1}, \ldots, A_{n}\right)$ by:

$$
A_{i}:= \begin{cases}A_{\left(Y \backslash Z_{1}\right) \cup Z_{1}} & \text { if } i \in C_{1} \\ A_{\left(Y \backslash Z_{2}\right) \cup Z_{2}} & \text { if } i \in C_{2} \\ A_{\left(Y \backslash Z_{3}\right) \cup Z_{3}} & \text { if } i \in C_{3} .\end{cases}
$$

By $C_{1} \notin \mathcal{W}$ and $N \backslash C_{1}=C_{2} \cup C_{3}$ we have $C_{2} \cup C_{3} \in \mathcal{W}$ by claim 1 , and so $Z_{1} \subseteq$ $F\left(A_{1}, \ldots, A_{n}\right)$. By $C \in \mathcal{W}$ and $C \subseteq C_{1} \cup C_{3}$ we have $C_{1} \cup C_{3} \in \mathcal{W}$ by claim 3 , and so $Z_{2} \subseteq F\left(A_{1}, \ldots, A_{n}\right)$. Further, $Z_{3} \subseteq F\left(A_{1}, \ldots, A_{n}\right)$ as $C_{1} \cup C_{2}=C^{*} \in \mathcal{W}$. Finally, $Y \backslash\left(Z_{1} \cup Z_{2} \cup Z_{3}\right) \subseteq F\left(A_{1}, \ldots, A_{n}\right)$ as $N \in \mathcal{W}$ by claim 1 . In summary, we have $Y \subseteq F\left(A_{1}, \ldots, A_{n}\right)$, violating consistency.

Claim 5. There is a dictator.

Consider the intersection of all winning coalitions, $\widetilde{C}:=\cap_{C \in \mathcal{W}} C$. By claim 4 , $\widetilde{C} \in \mathcal{W}$. So $\widetilde{C} \neq \emptyset$, as by claim $1 \emptyset \notin \mathcal{W}$. Consequently, there exists a $j \in \widetilde{C}$. I prove that $j$ is a dictator. Consider any $\left(A_{1}, \ldots, A_{n}\right) \in \operatorname{Domain}(F)$ and $p \in X$, and let us prove that $p \in F\left(A_{1}, \ldots, A_{n}\right)$ if and only if $p \in A_{j}$. If $p \in F\left(A_{1}, \ldots, A_{n}\right)$ then $C:=\left\{i: p \in A_{i}\right\} \in \mathcal{W}$; hence $j \in C$ (since $j$ belongs to every winning coalition), i.e. $p \in A_{j}$. If $p \notin F\left(A_{1}, \ldots, A_{n}\right)$, then $\neg p \in F\left(A_{1}, \ldots, A_{n}\right)$; so by an argument analogous to the one just used, $\neg p \in A_{j}$, whence $p \notin A_{j}$.

I now turn to Theorem $2^{*}$. Case 3 in the proof is essentially an argument made in Dokow and Holzman (2005).

Proof of Theorem $2^{*}$. Assume $n \geq 3$ and $X$ contains a contingent proposition. I show that if not all of the agenda conditions of asymmetry, $\left(\mathrm{i}^{*}\right)$ and (ii*) hold then there exists a regular, systematic and non-dictatorial aggregation rule $F$.

Case 1: $X$ is not asymmetric. Let $F$ be an inversely dictatorial rule, defined on the universal domain by $F\left(A_{1}, \ldots, A_{n}\right)=X \backslash A_{j}$ for some inverse dictator $j . F$ is obviously systematic and non-dictatorial and generates complete judgment sets. $F$ also generates consistent judgment sets, because $F\left(A_{1}, \ldots, A_{n}\right)=\left\{\neg p: p \in A_{j}\right\}$ is consistent as $X$ is not asymmetric.

Case 2: $X$ violates (i*). Let $F$ be majority rule among the persons $1,2,3$, defined on the universal domain by $F\left(A_{1}, \ldots, A_{n}\right)=\left\{p \in X\right.$ : at least two of the sets $A_{1}, A_{2}, A_{3}$ contains $p$. Obviously, $F$ is systematic and generates complete judgment sets. $F$ is also non-dictatorial since $x$ contains a contingent proposition (if $X$ contained only tautologies and contradictions, every $i$ would be a dictator). To show that $F$ generates consistent judgment sets, assume for contradiction that $Y:=F\left(A_{1}, \ldots, A_{n}\right)$ is inconsistent. For each $i \in\{1,2,3\}$, consider the subset $Z_{i}:=\left\{p \in Y: p \notin A_{i}\right\}$; we have $\left(Y \backslash Z_{i}\right) \cup Z_{i}^{\urcorner}=A_{i}$, which is consistent. Moreover, $Z_{1}, Z_{2}, Z_{3}$ are pairwise disjoint: if $p \in Z_{j} \cap Z_{k}$ (for distinct $j, k \in\{1,2,3\}$ ) then $p \notin A_{j}$ and $p \notin A_{k}$, and so $p \notin Y$ by definition of $F$, in contradiction with $Z_{j} \cap Z_{k} \subseteq Y$. But then (i*) would hold, a contradiction. So $Y$ is consistent. 
Case 3: $X$ violates (ii*). Define $F$ on the universal domain by $F\left(A_{1}, \ldots, A_{n}\right):=$ $\left\{p \in X\right.$ : the number of persons $i \in\{1,2,3\}$ with $p \in X_{i}$ is odd $\}$. Obviously, $F$ is systematic. $F$ is non-dictatorial for the same reason as in case $2 . \quad F$ also generates complete judgment sets, as $n$ is odd. Now assume for contradiction that $Y:=F\left(A_{1}, \ldots, A_{n}\right)$ is inconsistent. The sets $Z_{1}:=A_{1} \backslash\left(A_{2} \cup A_{3}\right), Z_{2}:=A_{2} \backslash\left(A_{1} \cup A_{3}\right)$ and $Z_{3}:=A_{3} \backslash\left(A_{1} \cup A_{2}\right)$ are pairwise disjoint. Further, for any distinct $j, k \in\{1,2,3\}$, letting $i \in\{1,2,3\} \backslash\{j, k\}$, we have $Z_{j} \cup Z_{k}=\left\{p \in Y: p \notin A_{i}\right\}=Y \backslash A_{i}$, and so

$$
\begin{aligned}
\left.\left(Y \backslash\left(Z_{j} \cup Z_{k}\right)\right) \cup\left(Z_{j} \cup Z_{k}\right)\right\urcorner & \left.=\left(Y \backslash\left(Y \backslash A_{i}\right)\right) \cup\left(Y \backslash A_{i}\right)\right\urcorner \\
& =\left(Y \cap A_{i}\right) \cup\left(A_{i} \backslash Y\right)=A_{i},
\end{aligned}
$$

a consistent set. But then (ii*) holds, a contradiction. So $Y$ is consistent.

\section{Judgment aggregation in general logics vs. in a par- ticular logic}

Not all results on judgment aggregation can or should be stated in general logics: some results require a particular logic, but often not classical propositional logic.

The general logics model is appropriate for results about agendas (decision problems) that are characterised by certain relations (of inconsistency or entailment) between the propositions, regardless of the particular logic generating these relations or the particular syntactic form of the propositions. Examples are the minimally connected agenda of Theorem 1, and, if suitably redefined in general logics, Nehring and Puppe's (2002) agendas, Gärdenfors' Boolean algebra agenda, and Dietrich's (2004) atomic agenda. ${ }^{33}$

By contrast, the restriction to a particular logic is needed for results about agendas (decision problems) characterised by propositions of a particular syntactic form, hence available only in particular languages L. I give two examples. Dietrich's (2005) possibility theorem holds for network agendas $X$, which belong to the conditional logic $C^{+}$and contain only propositions of the following syntactic forms (and their negations): atomic propositions, and (bi)conditionals $p \rightarrow q$ or $p \leftrightarrow q$, where $p$ and $q$ are conjunctions of one or more atomic propositions. Pauly and van Hees' (2004) Theorem 3 holds for atomically closed agendas $X$, which belong to classical propositional logic and contain (i) each atomic proposition $a$ that occurs in some proposition in $X$, and (ii) the propositions $a \wedge b, \neg a \wedge b, a \wedge \neg b, \neg a \wedge \neg b$ for any atomic propositions $a, b \in X$. It is, however, possible to restate their Theorem 3 for a

\footnotetext{
${ }^{33}$ For instance, the definition of atomic agendas can be restated as follows for a general logic $(\mathbf{L}, \vDash)$ (and Dietrich's 2004 impossibility result "Corollary 2" still holds if $(\mathbf{L}, \models)$ satisfies L1-L3). Intuitively, the atoms of $X$ are the "logically strongest" consistent members of $X$. For instance, if $X$ belongs to a classical propositional logic, contains only propositions made up of the (distinct) atomic propositions $a, b$, and contains $a \wedge b, a \wedge \neg b, \neg a \wedge b, \neg a \wedge \neg b$, then the latter four propositions are atoms of $X(a \wedge b$ is an atom as it entails each $q \in X$ consistent with $a \wedge b$ ). Formally, call $p \in X$ an atom of $X$ if $p$ is consistent and $p$ is inconsistent with a member of each pair $q, \neg q \in X$. Now $X$ is intuitively atomic if it is rich in atoms in the sense that the disjunction of all its atoms is a tautology (the previous example agenda is atomic as the disjunction of $a \wedge b, a \wedge \neg b, \neg a \wedge b, \neg a \wedge \neg b$ is a tautology) - but note that disjunctions need not be formable in $\mathbf{L}$ (especially if they are disjunctions of infinitely many atoms). However, as a disjunction of propositions is a tautology just in case the conjunction of the negations of these propositions is inconsistent, we can formally define $X$ as atomic if the set $\{\neg p: p$ is an atom of $X\}$ is inconsistent.
} 
general logic satisfying L1-L3, plus $\mathrm{L}_{\wedge}$ (see footnote 11) to ensure that conjunctions are formable. ${ }^{34}$

\section{Conclusion}

I have argued that a large variety of decision problems can be represented in logical terms, where each decision problem requires its particular logical language able to express the propositions under consideration. To be precise, two conditions must hold for a collective decision problem to be formalisable as a judgment aggregation problem in general logics (see Section 2.3):

(i) A decision can be construed as the acceptance or rejection of (usually interconnected) propositions from a possibly rich logical language, according to some relevant notion of collective acceptance/rejection (often belief; or desire, belief of moral desirability, act of making true, etc.).

(ii) The informational basis of a decision can be construed as the individuals' acceptances/rejections of these propositions, according to some relevant notion of individual acceptance/rejection (often belief).

For instance, Arrowian preference aggregation problems satisfy (i)-(ii), with propositions of the form $x R y$ in a predicate logic. While (i) often holds, (ii) fails whenever the informational basis takes a different form. Notably, the informational basis of welfare economics (in A. Sen's model of social welfare functionals) is richer than individual acceptances/rejections of ranking propositions $x R y$ : it consists of individual welfare functions. So welfare economics is not a special case of judgment aggregation.

Given the multitude of logics and their complexity, one might have feared that a separate approach is needed for each type of decision problem (each logic), and that judgment aggregation looses its unity and simplicity as a field. Fortunately, working with general logics (say, based on L1-L3) is simple and captures various logics (hence decision problems), notably classical propositional logic and many modal, conditional, predicate, and fuzzy logics. The framework L1-L3 (plus perhaps L5) is often appropriate when consistency and completeness are the only rationality conditions under consideration. Condition L4 (or weaker conditions) may become additionally useful to study the rationality condition of deductive closure.

To demonstrate that results can often be proven for a general (rather than a particular) logic, I have shown that many simple tools underlying the typical proofs in judgment aggregation are also available in general logics. I have also generalised existing impossibility theorems about systematic aggregation rules to general logics.

I hope to have convinced the reader that working with general logics does not make judgment aggregation more difficult, but far more general, and more transparent by removing unnecessary special assumptions on the logic.

\footnotetext{
${ }^{34}$ To do so, suppose there is a set $\mathcal{A} \subseteq \mathbf{L}$ of logically independent propositions, called the atomic propositions ("logically independent" means that any set containing exactly one member of each pair $p, \neg p \in \mathcal{A}$ is consistent). Then Pauly and van Hees' Theorem 3 remains true in L1-L3, $\mathrm{L}_{\wedge}$ if the definition of an atomically closed agenda is complemented by a third condition: each $p \in X$ is either an atomic proposition or constructed from atomic propositions using $\neg$ and $\wedge$.
} 


\section{References}

Bovens, L. and W. Rabinowicz (2004) Democratic Answers to Complex Questions - an Epistemic Perspective, Synthese, forthcoming.

Brennan, G. (2001) Collective Coherence? Int. Rev. Law Econ. 21(2): 197-211

Chapman, B. (1998) More Easily Done than Said: Rules, Reason and Rational Social Choice, Oxford Journal of Legal Studies 18: 293-329

Chapman, B. (2002) Rational Aggregation, Polit. Philos. Econ. 1(3): 337-354

Dietrich, F. (2004) Judgment Aggregation: (Im)possibility Theorems, J. Econ. Theory, forthcoming

Dietrich, F. (2005) The Possibility of Judgment Aggregation for Network Agendas, working paper, University of Konstanz

Dietrich, F. and C. List (2004a) Strategy-proof Judgment Aggregation, working paper, London School of Economics

Dietrich, F. and C. List (2004b) A Liberal Paradox for Judgment Aggregation, working paper, London School of Economics

Dietrich, F. and C. List (2005) Judgment Aggregation by Quota Rules, working paper, London School of Economics

Dokow, E. and R. Holzman (2005) Aggregation of Binary Relations, working paper, Technion Israel Institute of Technology

Gärdenfors, P. (2004) An Arrow-like Theorem for Voting with Logical Consequences, working paper, Lund University

Gekker, R. (2003) A Probability-based doxastic logic, working paper, Department of Economics, NUI, Galway, Ireland

Hintikka, J. (1971) Some Main Problems of Deontic Logic. In: Hilpinen, R. (1971) Deontic Logic: Introductory and Systematic Readings, Dordrecht, D. Reidel, p. $59-104$.

Kornhauser, L. A. and L. G. Sager (1986) Unpacking the Court, Yale Law Journal 96: $82-117$

Kripke, S. (1963) Semantical analysis of modal logic, I: normal propositional calculi, Zeitschrift für mathematische Logik und Grundlagen der Mathematik 9: 67-96

Lewis, D. (1973) Counterfactuals, Oxford: Basil Blackwell

List, C. (2003) A Possibility Theorem on Aggregation over Multiple Interconnected Propositions, Math. Soc. Sci. 45(1): 1-13

List, C. (2004a) The Probability of Inconsistencies in Complex Collective Decisions, Soc. Choice Welfare, forthcoming.

List, C. (2004b) A Model of Path Dependence in Decisions over Multiple Propositions, Amer. Polit. Sci. Rev. 98(3): 495-513

List, C. and P. Pettit (2002) Aggregating Sets of Judgments: An Impossibility Result, Econ. Philos. 18: 89-110

List, C. and P. Pettit (2004) Aggregating Sets of Judgments: Two Impossibility Results Compared, Synthese 140(1-2): 207-235

Mendelssohn, E. (1979) Introduction to Mathematical Logic. D. Van Nostrand

Mongin, P. (2005) Factoring out the Impossibility of Logical Aggregation, working paper, CNRS, Paris

Nehring, K. (2003) Arrow's Theorem as a Corollary, Economics Letters 80: 379382 
Nehring, K. and C. Puppe (2002) Strategy-Proof Social Choice on Single-Peaked Domains: Possibility, Impossibility and the Space Between, working paper, University of California at Davies

Nehring, K. and C. Puppe (2004) Consistent Judgement Aggregation: A Characterization, working paper, University of Karlsruhe

Pauly, M. and M. van Hees (2004) Logical Constraints on Judgment Aggregation, Journal of Philosophical Logic, forthcoming

Pettit, P. (2001) Deliberative Democracy and the Discursive Dilemma, Philosophical Issues 11: 268-299

Pigozzi, G. (2004) Collective Decision-Making without Paradoxes: An Argumentbased Account, working paper, King's College, London

Priest, G. (2001) An Introduction to Non-classical Logic, Cambridge Univ. Press

Stalnaker, R. (1968) A Theory of Conditionals, in N. Rescher (ed) Studies in Logical Theory, Blackwell: Oxford

van Hees, M. (2004) The Limits of Epistemic Democracy, working paper, University of Groningen

Wagner Decew, J. (1981) Conditional Obligation and Counterfactuals, Journal of Philosophical Logic 10(1): 55 - 72

Wilson R (1975) On the Theory of Aggregation. Journal of Economic Theory 10: 89-99 